\title{
Specific Synthesis and Reaction of Hetero- and Homobridged Diruthenium Carbonyl Complexes Containing One or Two $\mu$-Azolato Bridges
}

\author{
Kom-Bei Shiu,* Wei-Ming Lee, and Chen-Lan Wang \\ Department of Chemistry, National Cheng Kung University, \\ Tainan, Taiwan 701, Republic of China \\ Sue-Lein Wang and Fen-Ling Liao \\ Department of Chemistry, National Tsing Hua University, \\ Hsinchu, Taiwan 300, Republic of China \\ J u-Chun Wang and Lin-Shu Liou \\ Department of Chemistry, Soochow University, \\ Taipe, Taiwan 111, Republic of China \\ Shie-Ming Peng and Gene-Hsiang Lee \\ Department of Chemistry, National Taiwan University, \\ Taipe, Taiwan 106, Republic of China \\ Michael Y. Chiang \\ Department of Chemistry, National Sun Yat-Sen University, \\ Kaohsiung, Taiwan 804, Republic of China \\ Received February 5, $1996^{\otimes}$
}

\begin{abstract}
Diruthenium(I) carbonyl complexes with either hetero- or homobridges, $\left[\mathrm{Ru}_{2}(\mu-\mathrm{Pz})_{2}(\mathrm{CO})_{4^{-}}\right.$ $\left.(\mathrm{HPz})_{2}\right](\mathbf{1}),\left[\mathrm{Ru}_{2}\left(\mu-\mathrm{Pz}^{\prime}\right)\left(\mu-\mathrm{O}_{2} \mathrm{CMe}\right)(\mathrm{CO})_{4}\left(\mathrm{HPz}^{\prime}\right)_{2}\right](\mathbf{2})$, and $\left[\mathrm{Ru}_{2}\left(\mu-\mathrm{Pz}^{\prime}\right)_{2}(\mathrm{CO})_{4}\left(\mathrm{HPz}^{\prime}\right)_{2}\right](\mathbf{3})$, can be prepared specifically. These complexes reacted with either nucleophiles or electrophiles to produce selectively only one product. The terminal azole groups, pyrazole (HPz) or 3,5-

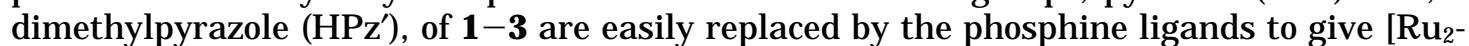
$\left.(\mu-\mathrm{Pz})_{2}(\mathrm{CO})_{4}\left(\mathrm{PPh}_{3}\right)_{2}\right](4),\left[\mathrm{Ru}_{2}\left(\mu-\mathrm{Pz} z^{\prime}\right)\left(\mu-\mathrm{O}_{2} \mathrm{CMe}\right)(\mathrm{CO})_{4}\left(\mathrm{PPh}_{3}\right)_{2}\right](5),\left[\mathrm{Ru}_{2}\left(\mu-\mathrm{Pz}^{\prime}\right)_{2}(\mathrm{CO})_{4}\left(\mathrm{PPh}_{3}\right)_{2}\right](6)$, and $\left[\mathrm{Ru}_{2}(\mu-\mathrm{Pz})_{2}(\mathrm{CO})_{4}\left(\eta^{1} \text {-dppm }\right)_{2}\right]$ (7). The $\mu$-acetato bridge is more fragile than the $\mu$-azolato bridge, and only the former bridge of $\mathbf{5}$ can be replaced by $\mathrm{Pz}^{-}$and $\mathrm{SR}^{-}$to afford $\left[\mathrm{Ru}_{2}(u-\right.$ $\left.\left.\mathrm{Pz}^{\prime}\right)(u-\mathrm{Pz})(\mathrm{CO})_{4}\left(\mathrm{PPh}_{3}\right)_{2}\right](\mathbf{8})$ and $\left[\mathrm{Ru}_{2}\left(\mu-\mathrm{Pz} \mathrm{z}^{\prime}\right)(\mu-\mathrm{SR})(\mathrm{CO})_{4}\left(\mathrm{PPh}_{3}\right)_{2}\right](\mathrm{R}=\mathrm{Ph}(\mathbf{9})$, tBu (10)). Heating the mixture of $\mathbf{3}$ with dppm in THF gave a product retaining all the $\mu$-azolato bridges but losing two carbonyls, $\left[\mathrm{Ru}_{2}(\mu-\mathrm{Pz})_{2}(\mathrm{CO})_{2}\left(\mu_{1}, \eta^{2} \text {-dppm }\right)_{2}\right]$ (11), whereas a similar reaction between 1-3 and nitrogen-bidentate ligands gave products retaining all four carbonyls but only one $\mu$-azolato bridge, $\left[\mathrm{Ru}_{2}(\mu-\mathrm{L})(\mu-\mathrm{CO})_{2}(\mathrm{CO})_{2}\left(\mu_{1}, \eta^{2}-(\mathrm{N}-\mathrm{N})\right)_{2}\right]^{+}\left(\mathrm{L}=\mathrm{Pz}, \mathrm{N}-\mathrm{N}=\right.$ bpy $\left([\mathbf{1 2}]^{+}\right)$, phen $\left([\mathbf{1 3}]^{+}\right) ; \mathrm{L}=\mathrm{Pz}, \mathrm{N}-\mathrm{N}=$ bpy $\left([\mathbf{1 4}]^{+}\right)$, phen $\left.\left([\mathbf{1 5}]^{+}\right)\right)$. The electrophilic addition of 4 with $\mathrm{I}_{2}$ produced $\left[\mathrm{Ru}_{2}(\mu-\mathrm{Pz})_{2}(\mu-\mathrm{I})(\mathrm{CO})_{4}\left(\mathrm{PPh}_{3}\right)_{2}\right]\left[\mathrm{I}_{3}\right](\mathbf{1 6})$. The X-ray structure of this product confirms the cleavage of the Ru-Ru bond rather than the $\mu$-azolato bridges. However, the $\mu$-azolato and -acetato bridges, as well as the terminal azole groups, of 1-6 can be easily removed by an electrophilc reagent such as $\mathrm{Et}_{3} \mathrm{O}^{+} \mathrm{BF}_{4^{-}}$in the presence of $\mathrm{MeCN}$ to give $\left[\mathrm{Ru}_{2}(\mathrm{CO})_{4^{-}}\right.$ $\left.(\mathrm{MeCN})_{6}\right]\left[\mathrm{BF}_{4}\right]_{2}$ and $\left[\mathrm{Ru}_{2}(\mathrm{CO})_{4}(\mathrm{MeCN})_{4}\left(\mathrm{PPh}_{3}\right)_{2}\right]\left[\mathrm{BF}_{4}\right]_{2}$.
\end{abstract}

\section{Introduction}

The coordination chemistry of the dirhodium and -iridium complexes has been well studied recently. Particularly, those containing the $\mu$-azolato bridging group are under intensive investigations, due to the ability of the apparently strong $\mu$-azolato bridge enabling to straddle an unusual range of intermetallic separations to hold two adjacent metal centers in chemically extremely stable configurations. ${ }^{1}$ In view of the rarely studied coordination chemistry of the diruthenium complexes with the $\mu$-azolato bridge, ${ }^{2}$ and the significance of the diruthenium(I) carbonyl complexes

\footnotetext{
${ }^{\otimes}$ Abstract published in Advance ACS Abstracts, May 15, 1996.
}

in being either involved as the active intermediates in homogeneously catalyzed reactions or catalytic precursors for the carbonylation of amines, the hydrogenation of carboxylic acids, and the addition of acetic acid to alkynes, ${ }^{3}$ we decided to explore the synthesis and reactivity some diruthenium(I) carbonyl complexes containing the $\mu$-azolato linkage.

In this paper, we present the following new information: (1) a convenient approach to prepare specifically both hetero- and homobridged diruthenium carbonyl complexes with one or two $\mu$-azolato linkages, (2) the selectivenucleophilic and el ectrophilic reactions of these complexes to give only one type of product, (3) the X-ray 
structures of eight representative reaction products, and (4) the novel propensity of the $\mu$-azolato linkage, especially the cleavable feature first found in the dimetal system.

\section{Results and Discussion}

Synthesis. Although complexes $\left[\mathrm{Ru}_{2}(\mu-\mathrm{L})_{2}(\mathrm{CO})_{6}\right](\mathrm{HL}$ $=$ pyrazole $(\mathrm{HPz})$, 3,5-dimethylpyrazole $\left(\mathrm{HPz}^{\prime}\right)$ ) were previously described to be important precursors to several other ruthenium derivatives, the synthetic approaches used suffer either a low yield2a,e or a tedious procedure. $^{2 c}$ As demonstrated below, new dinuclear species in the types of $\left[\mathrm{Ru}_{2}(\mu-\mathrm{L})_{2}(\mathrm{CO})_{4}(\mathrm{HL})_{2}\right]$ and $\left[\mathrm{Ru}_{2}(\mu-\right.$ $\left.\left.\mathrm{Pz}^{\prime}\right)\left(\mu-\mathrm{O}_{2} \mathrm{CMe}\right)(\mathrm{CO})_{4}\left(\mathrm{HPz}^{\prime}\right)_{2}\right]$ can be obtained specifically in satisfactory yield and employed as better precursors to a variety of other $\mathrm{Ru}(\mathrm{I})$ and $\mathrm{Ru}(\mathrm{II})$ derivatives. Importantly, all the conversions appear to follow selective pathways.

In the presence of $\mathrm{Et}_{3} \mathrm{~N}$, catena- $\left[\mathrm{Ru}\left(\mathrm{O}_{2} \mathrm{CMe}\right)(\mathrm{CO})_{2}\right]$ reacted with excess $\mathrm{HPz}$ or $\mathrm{HPz}$ in EtOH gave only one diruthenium derivative with either homo- or heterobridges, $\left[\mathrm{Ru}_{2}(\mu-\mathrm{Pz})_{2}(\mathrm{CO})_{4}(\mathrm{HPz})_{2}\right](\mathbf{1})$ and $\left[\mathrm{Ru}_{2}\left(\mu-\mathrm{Pz}^{\prime}\right)(\mu-\right.$ $\left.\left.0_{2} \mathrm{CMe}\right)(\mathrm{CO})_{4}\left(\mathrm{HPz}^{\prime}\right)_{2}\right]$ (2), respectively. The homogridged compounds 1 can be obtained alternatively from 触e reaction of $\left[\mathrm{Ru}_{2}(\mathrm{CO})_{4}(\mathrm{MeCN})_{6}\right]\left[\mathrm{BF}_{4}\right]_{2}$ with $\mathrm{HPz} / \mathrm{Et}_{3} \mathrm{~N}$ a end $\mathrm{H}_{2} \mathrm{O}$. Complex $\left[\mathrm{Ru}_{2}\left(\mu-\mathrm{Pz}^{\prime}\right)_{2}(\mathrm{CO})_{4}\left(\mathrm{HPz}^{\prime}\right)_{2}\right](3)$ can be (1) (a) Uson, R.; Oro, L. A.; Ciriano, M. A.; Pinillos, M. T. J. \& Organomet. Chem. 1981, 205, 247. (b) Beveridge, K. A.; Bushnell, G. I $\dot{H}$.; Dixon, K. R.; Eadie, D. T.; Stobart, S. R.; Atwood, J . L.; Zaworotko, $\geq \mathbb{Q}$ J.J. Am. Chem. Soc. 1982, 104, 920. (c) Coleman, A. W.; Eadie, D. E. Stobart, S. R.; Zaworotko, M. J .; Atwood, J. L. J. Am. Chem. Soc. 1982, 104, 922. (d) Bushnell, G. W.; Fjeldsted, O. K.; Stobart, S. R.; Z Zaworotko, M. J.J . Chem. Soc., Chem. Commun. 1983, 580. (e) Beveridge, K. A.; Bushnell, G. W.; Stobart, S. R.; Atwood, J. L.; 勇worotko, M. J. Organometallics, 1983, 2, 1447. (f) Bushnell, G. W. Stobart, S. R.; Vefghi, R.; Zaworotko, M. J .J. Chem. Soc., Chem. Gommun. 1984, 282. (g) Atwood, J. L.; Beveridge, K. A.; Bushnell, G. W. Z Ğem. 1984, 23, 4050. (h) Bushnell, G. W.; Fjeldsted, D. O. K.; Stobart, \& R.; Zaworotko, M.J .; Knox, S. A. R.; Macpherson, K. A. Organome $\varangle$ allics 1985, 4, 1107. (i) Bushnell, G. W.; Decker, M. J .; Eadie, D. T.; Z Stobart, S. R.; Vefghi, R.; Atwood, J . L.; Zaworotko, M. J . Organome ô tallics 1985, 4, 2106. (j) Claver, C.; Kalck, P.; Ridmy, M.; Thorez, A.; Z Gro, L. O.; Pinillos, M. T.; Apreda, M. C.; Cano, F. H.; Foces-Foces, C. If Chem. Soc., Dalton Trans. 1988, 1523. (k) Caromna, D.; Oro, L. O.; Ferez, P. L.; Tiripicchio, A.; Tiripicchio-Camellini, M. J . Chem. Soc.,

Talton Trans. 1989, 1427. (I) Pinillos, M. T.; Elduque, A.; Oro, L. A. Forg. Chim. Acta 1990, 178, 179. (m) Pinillos, M. T.; Elduque, A.; Oro, A.; Lahoz, F. J .; Bonati, F.; Tiripicchio, A.; Tiripicchio-Camellini, 两.J . Chem. Soc., Dalton Trans. 1990, 989. (n) Pinillos, M. T.; Elduque, A.; Lopez, J . A.; Lahoz, F. J .; Oro, L. A. J . Chem. Soc., Dalton Trans. 1991, 1391. (o) Carmona, D.; Ferrer, J .; Lahoz, F.J .; Oro, L. A.; Reyes, J .; Esteban, M. J . Chem. Soc., Dalton T'rans. 1991, 2811. (p) Carmona, D.; Ferrer, I .; Mendoza, A.; Lahoz, F. J .; Reyes, J .; Oro, L. A. Angew. Chem., Int. Ed. Engl. 1991, 30, 1171. (q) Pinillos, M. T.; Elduque, A.; Oro, L. A. Polyhedron 1992, 11, 1007. (r) Ciriano, M. A.; Tena, M. A.; Oro, L. A. J . Chem. Soc., Dalton Trans. 1992, 2123. (s) Pinillos, M. T.; Elduque, A.; Lopez, J . A.; Lahoz, F.J .; Oro, L. A.; Mann, B. E.J . Chem. Soc., Dalton Trans. 1992, 2389. (t) Tejel, C.; Ciriano, M. A.; Oro, L. A.; Tiripicchio, A.; U gozzoli, F. Organometallics 1994, 13, 4153.

(2) (a) Cabeza, J. A.; Landazuri, C.; Oro, L. A.; Tiripicchio, A. Tiripicchio-Camellini, M. J . Organomet. Chem. 1987, 322, C16. (b) Sherlock, S. J .; Cowie, M.; Singleton, E.; Steyn, M. M. d. V. J. Organomet. Che̋m. 1989, 361, 353. (c) Neumann, F.; Süss-Fink, G. J. Organomet. Chem. 1989, 367, 175. (d) Neumann, F.; Stoeckli-Evans, H.; Süss-Fink, G. J . Organomet. Chem. 1989, 379, 151. (e) Cabeza, J. A.; Landazuri, C.; Oro, L. A.; Belletti, D.; Tiripicchio, A.; TiripicchioCamellini, M. J . Chem. Soc., Dalton Trans. 1989, 1093.

(3) (a) Bianchi, M.; Menchi, G.; Francalanci, F.; Piacenti, F.; Matteoli, U.; Frediani, P. J . Organomet. Chem. 1980, 188, 109. (b) Bianchi, M.; Iani, P. F.; Matteoli, U.; Menchi, G.; Piacenti, F.; Petrucci, G. J . Organomet. Chem. 1983, 259, 207. (c) Suss-Fink, G.; Hermann G.; Morys, P.; Ellermann, J .; Veit, A. J . Organomet. Chem. 1985, 284, 263. (d) Matteoli, U.; Menchi, G.; Frediani, P.; Bianchi, M.; Piacenti, F. J . Organomet. Chem. 1985, 285, 281. (e) Kalck, P.; Sianti, M.; J enck, J .; Peyrille, B.; Peres, Y. J . Mol. Catal. 1991, 67, 19. (f) Frediani, P.; Bianchi, M.; Salvini, A.; Guarducci, R.; Carluccio, L. C.; Piacenti, F. J . Organomet. Chem. 1995, 498, 187.

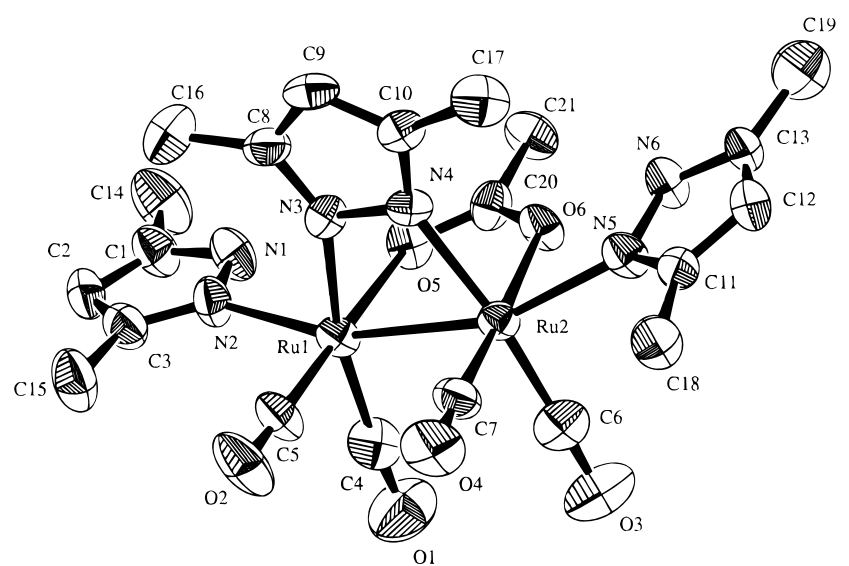

Figure 1. ORTEP plot of $\mathbf{2}$ with $50 \%$ thermal ellipsoids.

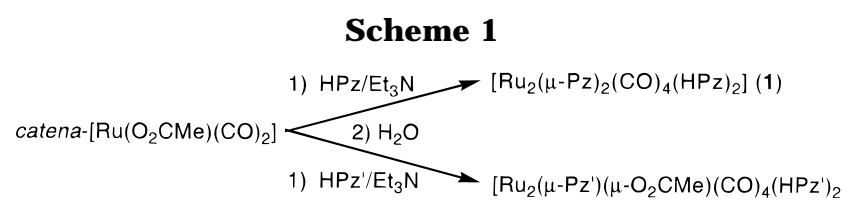

(2)

$\left[\mathrm{Ru}_{2}(\mathrm{CO})_{4}(\mathrm{MeCN})_{6}\right]\left[\mathrm{BF}_{4}\right]_{2} \frac{1) \mathrm{HPz}^{\prime} / \mathrm{Et}_{3} \mathrm{~N}}{2) \mathrm{H}_{2} \mathrm{O}}\left[\mathrm{Ru}_{2}\left(\mu-\mathrm{Pz}^{\prime}\right)_{2}(\mathrm{CO})_{4}\left(\mathrm{HPz}^{\prime}\right)_{2}\right](3)$

obtained likewise. The reactions involve obvi ously first the substitution of $\mathrm{MeCN}$ in $\left[\mathrm{Ru}_{2}(\mathrm{CO})_{4}(\mathrm{MeCN})_{6}\right]\left[\mathrm{BF}_{4}\right]_{2}$ with a better $\sigma$-donor, $\mathrm{L}^{-}$, via $\mathrm{HL} / \mathrm{Et}_{3} \mathrm{~N}$ to give $\left[\mathrm{Ru}_{2}\left(\mu, \eta^{2}-\right.\right.$ $\left.\mathrm{L})_{2}(\mathrm{CO})_{4}\left(\eta^{1}-\mathrm{L}\right)\right]^{2-}$ and then protonation with $\mathrm{H}_{2} \mathrm{O}$ to give $\mathbf{1}$ and $\mathbf{3}$ (Scheme 1). The specific formation of $\mathbf{2}$ rather than a mixture of $\mathbf{2}$ and $\mathbf{3}$ reflects probably that the nucleophilic substitution is stepwise and the steric hindrance of $\mu-\mathrm{Pz}^{\prime}$ in $\mathbf{2}$ may inhibit a subsequent replacement of the remaining $\mu$-acetato group by a second $\mathrm{Pz}^{-}$.

Compound $\mathbf{2}$ was structurally characterized (Figure 1). The heterobridged feature and the two $\mathrm{HPz}^{\prime}$ groups ligated to Ru at the axial sites were confirmed. The Ru-Ru distance of 2.682(1) $\AA$ in $\mathbf{2}$ (Table 1 ) is significantly shorter than that of $2.705(2) \AA$ in $\left[\mathrm{Ru}_{2}\left(\eta^{2}-\mathrm{Pz}^{\prime}\right)_{2^{-}}\right.$ $\left.(\mathrm{CO})_{6}\right]_{\text {. }}^{2 a, e}$ The longer distance may be due to a combination of both el ectronic and steric factors. The higher trans influence of the axial carbonyls in this compound, compared with that of the axial HPz' groups in $\mathbf{2}$, and the larger nonbonded interactions between two bulky $\mu-\mathrm{Pz}^{\prime}$ groups and other groups in $\left[\mathrm{Ru}_{2}\left(\eta^{2}-\mathrm{Pz}^{\prime}\right)_{2}(\mathrm{CO})_{6}\right]$, compared with those between one such $\mu-\mathrm{Pz}$ group and other groups in $\mathbf{2}$, may contribute to the Ru-Ru elongation.

Reactions with Nucleophiles. The two axial groups in either $\left[\mathrm{Ru}_{2}\left(\eta^{2}-\mathrm{L}\right)_{2}(\mathrm{CO})_{6}\right]^{2 \mathrm{e}}$ or $\left[\mathrm{Ru}_{2}(\mathrm{CO})_{4}(\mathrm{MeCN})_{6}\right]^{2+} 4 a$ were previously reported to be substitution labile. Complexes 1-3 behave similarly. The two axial HL groups of 1-3 are easily replaced by phosphine groups to give $\left[\mathrm{Ru}_{2}(\mu-\mathrm{Pz})_{2}(\mathrm{CO})_{4}\left(\mathrm{PPh}_{3}\right)_{2}\right](\mathbf{4}),\left[\mathrm{Ru}_{2}\left(\mu-\mathrm{Pz}^{\prime}\right)\left(\mu-\mathrm{O}_{2^{-}}\right.\right.$ $\left.\mathrm{CMe})(\mathrm{CO})_{4}\left(\mathrm{PPh}_{3}\right)_{2}\right]$ (5), $\left[\mathrm{Ru}_{2}\left(u-\mathrm{Pz}^{\prime}\right)_{2}(\mathrm{CO})_{4}\left(\mathrm{PPh}_{3}\right)_{2}\right]$ (6), and $\left[\mathrm{Ru}_{2}(\mu-\mathrm{Pz})_{2}(\mathrm{CO})_{4}\left(\eta^{1} \text {-dppm }\right)_{2}\right]$ (7) (Scheme 2) with $\mathbf{5}$ structurally characterized to confirm the heterobridged feature (Figure 2 ). The Ru-Ru distance increases from 2.682(1) $\AA$ in $\mathbf{2}$ to $2.7261(9) \AA$ in 5, apparently due to the increased nonbonded interactions between the bulkier $\mathrm{PPh}_{3}$ groups and other groups in the molecule.

(4) (a) Klemperer, W. G.; Zhong, B. Inorg. Chem. 1993, 32, 5821. (b) Shiu, K.-B.; Li, C.-H.; Chan, T.-J .; Peng, S.-M.; Cheng, M.-C.; Wang, S.-L.; Liao, F.-L.; Chiang, M. Y. Organometallics 1995, 14, 524. 


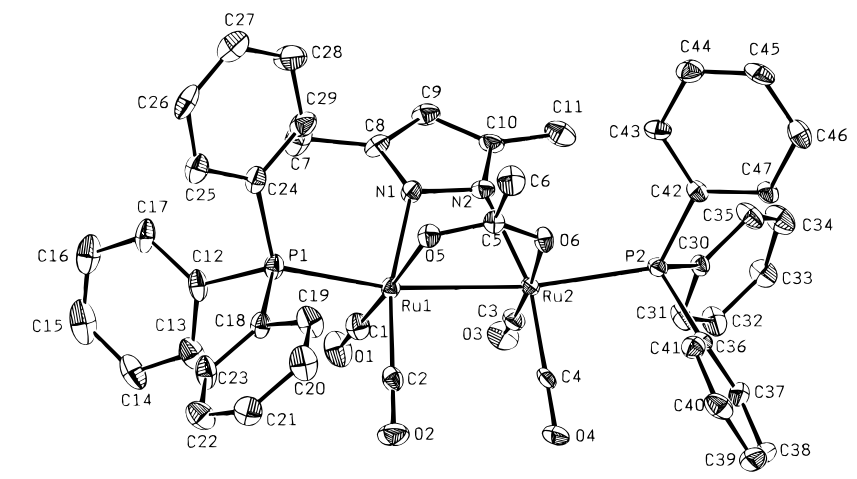

Figure 2. ORTEP plot of $\mathbf{5}$ with $50 \%$ probability ellipsoids.

Scheme 2
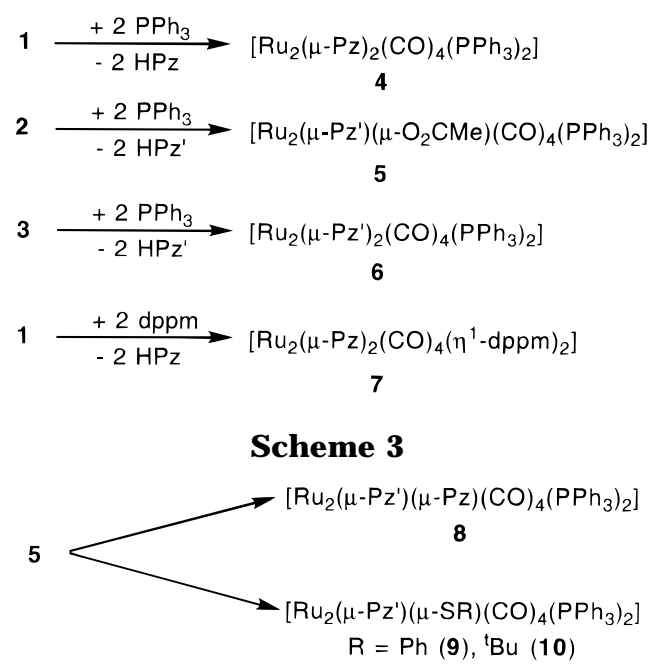

${ }^{31} \mathrm{P}\left\{{ }^{1} \mathrm{H}\right\} \mathrm{NMR}$ spectrum of 7 , like that of $\left[\mathrm{Ru}_{2}(\mu\right.$ $\left.\left.\oint_{2} \mathrm{CMe}\right)_{2}(\mathrm{CO})_{4}\left(\eta^{1} \text {-dppm }\right)_{2}\right]_{,}^{5}$ appears as two sets of mul-

tiplets at $\delta 12.12$ and -26.18 ppm consistent with an

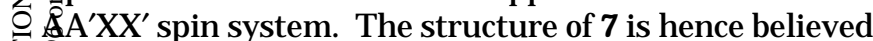
to be similar to that of 5 .

is The $\mu$-acetato bridge, rather than the $\mu$-Pz' bridge, in 5 can be replaced further by any smaller or stronger anionic $\sigma$-donor, like $\mathrm{Pz}^{-}$or thiolate anion, to give $\left[\mathrm{Ru}_{2^{-}}\right.$ ( (OO) $\left.{ }_{4}\left(\mathrm{PPh}_{3}\right)_{2}\right](\mathrm{R}=\mathrm{Ph}(\mathbf{9})$, tBu (10)) (Scheme 3), respecEvely. The ${ }^{31} \mathrm{P}\left\{{ }^{1} \mathrm{H}\right\}$ NMR spectrum of $\mathbf{1 0}$ appears as two doublets indicating probably the asymmetric orientation of either two $\mathrm{PPh}_{3}$ groups and/or the thiolate bridge with respect to the $\mathrm{Ru}-\mathrm{Ru}$ bond. $\mathbf{1 0}$ may be rigid in solution at room temperature on the NMR time scale. The single-crystal structure of $\mathbf{1 0}$ was thus carried out (Figure 3a). It reveals two asymmetrical $\mathrm{PPh}_{3}$ groups (Figure $3 b$ ) with the $\mathrm{P}(2)$ group at the axial site and the $P(1)$ group at the equatorial site, probably alleviating the repulsive nonbonded interactions if both groups are at the axial sites. The $R u-R u$ distance is 2.7469(5) A.

The reaction product between $\left[\mathrm{Ru}_{2}\left(\mu-\mathrm{Pz}^{\prime}\right)_{2}(\mathrm{CO})_{6}\right]$ and excess dppm was previously reported by Süss-Fink's group to be $\left[\mathrm{Ru}_{2}\left(\mu-\mathrm{Pz} z_{2}\right)_{2}(\mathrm{CO})_{4}\left(\eta^{1}-\mathrm{dppm}\right)_{2}\right]$, a formula similar to 7, but an unusal structure was suggested to have two $\eta^{1}$-dppm and one $\mathrm{CO}$ groups at one Ru atom and three $\mathrm{CO}$ groups at the other metal atom, probably on the basis of a typical $A A^{\prime} X X^{\prime}$ spin system observed

(5) Sherlock, S. J .; Cowie, M.; Singleton, E.; Steyn, M. M. d. V. Organometallics 1988, 7, 1663.
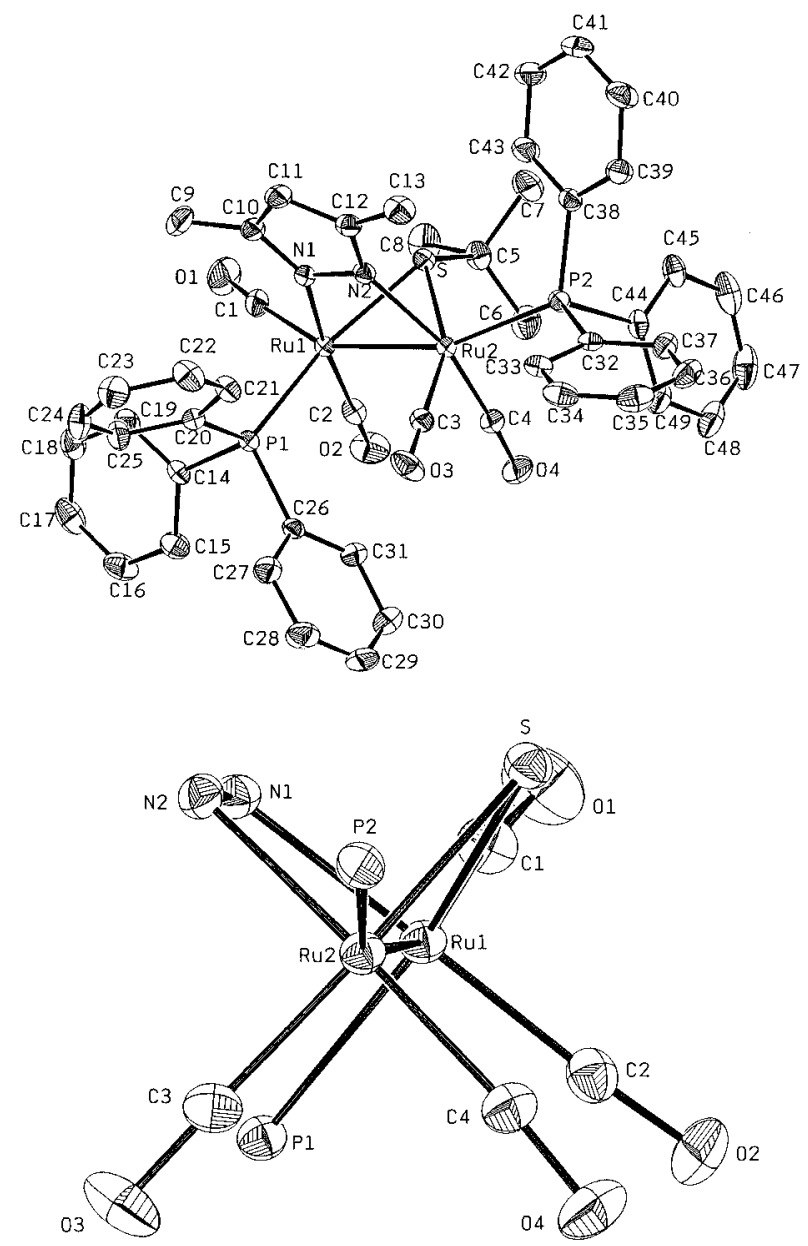

Figure 3. (a) Top: ORTEP plot of $\mathbf{1 0}$ with $50 \%$ thermal ellipsoids. (b) Bottom: ORTEP plot of $\mathbf{1 0}$ along the RuRu axis with phenyl and tert-butyl groups omitted.

in a ${ }^{31} \mathrm{P}\left\{{ }^{1} \mathrm{H}\right\}$ NMR spectrum. ${ }^{2 \mathrm{~d}}$ However, such a suggestion was questioned, on the basis of our findings that the $\mu-P z^{\prime}$ linkage is rather strong and resistant even with respect to a strong nucleophile such as a thiolate anion and that the axial groups in $\left[\mathrm{Ru}_{2}\left(\eta^{2}-\mathrm{L}\right)_{2}(\mathrm{CO})_{6}\right]$, $\left[\mathrm{Ru}_{2}(\mathrm{CO})_{4}(\mathrm{MeCN})_{6}\right]^{2+}$, or $\mathbf{1 - 3}$ are substitution Iabile. In order to obtain a clear-cut conclusion, we attempted a similar substitution reaction by following their reaction condition, i.e, by heating a mixture of $\mathbf{3}$ and excess dppm in THF for more than $24 \mathrm{~h}$. The compound we obtained shows very similar IR and ${ }^{1} \mathrm{H}-\mathrm{NMR}$ spectral data to those reported by Süss-Fink's group. It also exhibits two multiplets consistent with an $A^{\prime} X^{\prime} X^{\prime}$ spin system at $\delta 11.41$ and -30.26 , but these two values differ about 3.6-4.0 ppm from those reported. F urther, the elemental analysis results clearly indicate that the compound should not be formulated as $\left[\mathrm{Ru}_{2}\left(\mathrm{Pz} \mathrm{z}_{2}(\mathrm{CO})_{4^{-}}\right.\right.$ $\left.(\mathrm{dppm})_{2}\right]$ but $\left[\mathrm{Ru}_{2}\left(\mathrm{Pz}^{\prime}\right)_{2}(\mathrm{CO})_{2}(\mathrm{dppm})_{2}\right]$ (11) with only two, rather than four, carbonyls suggested. The crystal structure of $\mathbf{1 1}$ was hence determined to support this formulation. As shown in the simplified ORTEP plot of $\mathbf{1 1}$ (Figure 4), each Ru atom of $\mathbf{1 1}$ has one chelate dppm and one CO. The two carbonyls, $\mathrm{C}(1) \mathrm{O}(1)$ and $\mathrm{C}(2) \mathrm{O}(2)$, are in a gauche conformation with the torsion angle $\angle C(1)-R u(1)-R u(2)-C(2)=85.9^{\circ}$. Obviously the bidentate ligand dppm can replace only the axial $\mathrm{HPz}^{\prime}$ and carbonyl groups rather than cleave one or two apparently strong $\mu$-Pz' linkages in 3 (Scheme 4). 
Table 1. Selected Bond Lengths ( $(\AA)$ and Angles (deg)

\begin{tabular}{|c|c|c|c|c|c|c|c|}
\hline \multicolumn{8}{|c|}{ Bond Lengths for $\mathbf{2}$} \\
\hline $\mathrm{Ru}(1)-\mathrm{Ru}(2)$ & $2.682(1)$ & $\mathrm{Ru}(2)-\mathrm{O}(6)$ & $2.170(5)$ & $\mathrm{Ru}(1)-\mathrm{N}(2)$ & $2.261(7)$ & $\mathrm{C}(4)-\mathrm{O}(1)$ & $1.17(1)$ \\
\hline $\mathrm{Ru}(1)-\mathrm{N}(3)$ & $2.111(7)$ & $\mathrm{C}(5)-\mathrm{O}(2)$ & $1.135(10)$ & $\mathrm{Ru}(1)-\mathrm{C}(5)$ & $1.846(10)$ & $\mathrm{C}(6)-\mathrm{O}(3)$ & $1.160(10)$ \\
\hline $\mathrm{Ru}(1)-\mathrm{C}(4)$ & $1.82(1)$ & $\mathrm{C}(7)-\mathrm{O}(4)$ & $1.155(10)$ & $\mathrm{Ru}(1)-\mathrm{O}(5)$ & $2.170(6)$ & $\mathrm{C}(20)-\mathrm{O}(5)$ & $1.278(10)$ \\
\hline $\mathrm{Ru}(2)-\mathrm{N}(4)$ & $2.103(7)$ & $C(20)-O(6)$ & $1.252(10)$ & $R u(2)-N(5)$ & $2.213(7)$ & $N(1)-N(2)$ & $1.362(9)$ \\
\hline $\mathrm{Ru}(2)-C(6)$ & $1.839(10)$ & $N(3)-N(4)$ & $1.376(8)$ & $\mathrm{Ru}(2)-\mathrm{C}(7)$ & $1.850(10)$ & $N(5)-N(6)$ & $1.341(9)$ \\
\hline \multicolumn{8}{|c|}{ Bond Angles for $\mathbf{2}$} \\
\hline $\mathrm{Ru}(2)-\mathrm{Ru}(1)-\mathrm{N}(2)$ & $162.2(2)$ & $\mathrm{O}(6)-\mathrm{Ru}(2)-\mathrm{C}(7)$ & $178.1(3)$ & $\mathrm{Ru}(1)-\mathrm{Ru}(2)-\mathrm{N}(5)$ & $160.0(2)$ & $C(7)-R u(2)-C(6)$ & $88.1(4)$ \\
\hline $\mathrm{Ru}(2)-\mathrm{Ru}(1)-\mathrm{O}(5)$ & $84.3(2)$ & $C(6)-R u(2)-N(4)$ & 169.5(3) & $\mathrm{Ru}(1)-\mathrm{Ru}(2)-\mathrm{O}(6)$ & 83.7(1) & $\mathrm{Ru}(1)-\mathrm{C}(4)-\mathrm{O}(1)$ & 176.5(9) \\
\hline $\mathrm{O}(5)-\mathrm{C}(20)-\mathrm{O}(6)$ & $123.6(8)$ & $\mathrm{Ru}(1)-\mathrm{C}(5)-\mathrm{O}(2)$ & 179.0(8) & $O(5)-R u(1)-C(5)$ & 178.6(3) & $\mathrm{Ru}(2)-\mathrm{C}(6)-\mathrm{O}(3)$ & 178.3(9) \\
\hline$C(5)-R u(1)-C(4)$ & $92.1(3)$ & $\mathrm{Ru}(2)-\mathrm{C}(7)-\mathrm{O}(4)$ & $178.9(9)$ & $\mathrm{C}(4)-\mathrm{Ru}(1)-\mathrm{N}(3)$ & $167.9(3)$ & & \\
\hline \multicolumn{8}{|c|}{ Bond Lengths for $\mathbf{5}$} \\
\hline $\operatorname{Ru}(1)-\operatorname{Ru}(2)$ & $2.7261(9)$ & $\mathrm{Ru}(2)-\mathrm{O}(6)$ & $2.136(5)$ & $\mathrm{Ru}(1)-\mathrm{C}(1)$ & $1.834(9)$ & $C(5)-O(5)$ & $1.265(9)$ \\
\hline $\mathrm{Ru}(1)-\mathrm{N}(1)$ & $2.129(6)$ & $C(5)-O(6)$ & $1.246(8)$ & $\mathrm{Ru}(1)-\mathrm{O}(5)$ & $2.142(5)$ & $\mathrm{C}(1)-\mathrm{O}(1)$ & $1.142(11)$ \\
\hline $\mathrm{Ru}(1)-\mathrm{C}(2)$ & $1.871(8)$ & $\mathrm{C}(2)-\mathrm{O}(2)$ & $1.134(9)$ & $\mathrm{Ru}(2)-\mathrm{N}(2)$ & $2.127(6)$ & $\mathrm{C}(3)-\mathrm{O}(3)$ & $1.147(10)$ \\
\hline $\mathrm{Ru}(2)-\mathrm{P}(2)$ & $2.4324(21)$ & $\mathrm{C}(4)-\mathrm{O}(4)$ & $1.140(9)$ & $\mathrm{Ru}(2)-\mathrm{C}(4)$ & $1.865(7)$ & $N(1)-N(2)$ & $1.379(8)$ \\
\hline $\mathrm{Ru}(2)-\mathrm{C}(3)$ & $1.828(8)$ & $\mathrm{Ru}(1)-\mathrm{P}(1)$ & $2.4357(21)$ & & & & \\
\hline \multicolumn{8}{|c|}{ Bond Angles for $\mathbf{5}$} \\
\hline $\mathrm{Ru}(2)-\mathrm{Ru}(1)-\mathrm{P}(1)$ & 166.35(7) & $\mathrm{O}(6)-\mathrm{Ru}(2)-\mathrm{C}(3)$ & $174.8(3)$ & $\mathrm{Ru}(1)-\mathrm{Ru}(2)-\mathrm{P}(2)$ & 167.04(6) & $C(3)-R u(2)-C(4)$ & $92.4(3)$ \\
\hline $\mathrm{Ru}(1)-\mathrm{Ru}(2)-\mathrm{O}(6)$ & $83.66(13)$ & $C(4)-R u(2)-N(2)$ & $164.8(3)$ & $\mathrm{Ru}(2)-\mathrm{Ru}(1)-\mathrm{O}(5)$ & 82.94(13) & $\mathrm{Ru}(1)-\mathrm{C}(1)-\mathrm{O}(1)$ & $178.8(7)$ \\
\hline $\mathrm{O}(5)-\mathrm{C}(5)-\mathrm{O}(6)$ & $125.1(7)$ & $\mathrm{Ru}(1)-\mathrm{C}(2)-\mathrm{O}(2)$ & 179.2(8) & $\mathrm{O}(5)-\mathrm{Ru}(1)-\mathrm{C}(1)$ & $176.0(3)$ & $\mathrm{Ru}(2)-\mathrm{C}(3)-\mathrm{O}(3)$ & $175.8(7)$ \\
\hline$C(1)-R u(1)-C(2)$ & $89.8(4)$ & $\mathrm{Ru}(2)-\mathrm{C}(4)-\mathrm{O}(4)$ & $177.7(8)$ & $\mathrm{C}(2)-\mathrm{Ru}(1)-\mathrm{N}(1)$ & $166.4(3)$ & & \\
\hline \multicolumn{8}{|c|}{ Bond Lengths for $\mathbf{1 0}$} \\
\hline$R u(1)-R u(2)$ & $2.7469(5)$ & $\mathrm{Ru}(2)-\mathrm{N}(2)$ & $2.153(3)$ & $\mathrm{Ru}(1)-\mathrm{P}(1)$ & $2.3735(10)$ & $\mathrm{Ru}(2)-\mathrm{C}(3)$ & $1.871(4)$ \\
\hline$\cong \mathrm{Ru}(1)-\mathrm{S}$ & $2.3795(10)$ & $\mathrm{Ru}(2)-\mathrm{C}(4)$ & $1.859(4)$ & $\mathrm{Ru}(1)-\mathrm{N}(1)$ & $2.095(3)$ & $N(1)-N(2)$ & $1.375(4)$ \\
\hline$\delta \mathrm{Ru}(1)-\mathrm{C}(1)$ & $1.906(4)$ & $\mathrm{C}(1)-\mathrm{O}(1)$ & $1.136(6)$ & $\mathrm{Ru}(1)-\mathrm{C}(2)$ & $1.865(4)$ & $\mathrm{C}(2)-\mathrm{O}(2)$ & $1.144(5)$ \\
\hline$R u(2)-P(2)$ & $2.4261(10)$ & $\mathrm{C}(3)-\mathrm{O}(3)$ & $1.146(5)$ & $\mathrm{Ru}(2)-\mathrm{S}$ & $2.4276(10)$ & $\mathrm{C}(4)-\mathrm{O}(4)$ & $1.139(5)$ \\
\hline \multicolumn{8}{|c|}{ Bond Angles for $\mathbf{1 0}$} \\
\hline तु $u(2)-R u(1)-P(1)$ & 103.54(3) & $\mathrm{Ru}(1)-\mathrm{Ru}(2)-\mathrm{C}(4)$ & $92.43(12)$ & $\mathrm{Ru}(1)-\mathrm{Ru}(2)-\mathrm{P}(2)$ & & $C(3)-R u(2)-C(4)$ & 87.34 \\
\hline Ru(1)-S-Ru(2) & $69.69(3)$ & $N(2)-R u(2)-C(4)$ & $162.97(4)$ & $C(1)-R u(1)-C(2)$ & $88.32(18)$ & $\mathrm{Ru}(1)-\mathrm{C}(1)-\mathrm{O}(1)$ & $171.2(4)$ \\
\hline Ru(2)-Ru(1)-C(1) & $154.94(12)$ & $\mathrm{Ru}(1)-\mathrm{C}(2)-\mathrm{O}(2)$ & $176.0(4)$ & $\operatorname{Ru}(2)-\operatorname{Ru}(1)-C(2)$ & $102.05(12)$ & $\mathrm{Ru}(2)-\mathrm{C}(3)-\mathrm{O}(3)$ & 179.2(4) \\
\hline$\ddot{\sigma}(2)-R u(1)-N(1)$ & $172.98(15)$ & $\mathrm{Ru}(2)-\mathrm{C}(4)-\mathrm{O}(4)$ & $175.5(4)$ & $\mathrm{Ru}(1)-\mathrm{Ru}(2)-\mathrm{C}(3)$ & $102.52(12)$ & & \\
\hline \multicolumn{8}{|c|}{ Bond Lengths for $\mathbf{1 1}$} \\
\hline : $\mathrm{Ru}(1)-\mathrm{Ru}(2)$ & $2.738(1)$ & $\mathrm{Ru}(2)-\mathrm{N}(2)$ & $2.120(3)$ & $\mathrm{Ru}(1)-\mathrm{P}(3)$ & $2.416(1)$ & $\mathrm{Ru}(2)-\mathrm{N}(4)$ & $2.169(3)$ \\
\hline $R u(1)-P(4)$ & $2.309(1)$ & $\mathrm{Ru}(2)-\mathrm{C}(2)$ & $1.828(4)$ & $\mathrm{Ru}(1)-\mathrm{N}(1)$ & $2.136(3)$ & $\mathrm{C}(1)-\mathrm{O}(1)$ & $1.150(5)$ \\
\hline 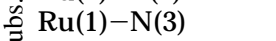 & $2.112(3)$ & $\mathrm{C}(2)-\mathrm{O}(2)$ & $1.168(5)$ & $\mathrm{Ru}(1)-\mathrm{C}(1)$ & $1.846(4)$ & $N(1)-N(2)$ & $1.368(4)$ \\
\hline $\mathrm{Ru}(2)-\mathrm{P}(1)$ & $2.386(1)$ & $N(3)-N(4)$ & $1.371(4)$ & $\mathrm{Ru}(2)-\mathrm{P}(2)$ & $2.316(1)$ & & \\
\hline \multicolumn{8}{|c|}{ Bond Angles for $\mathbf{1 1}$} \\
\hline$\nexists R u(2)-R u(1)-P(3)$ & 175.6(1) & $P(1)-R u(2)-P(2)$ & $72.0(1)$ & $P(3)-R u(1)-P(4)$ & 72.3(1) & $P(2)-R u(2)-N(2)$ & 177.5(1) \\
\hline$\stackrel{P}{(4)-R u(1)-N(3)}$ & $176.1(1)$ & $C(2)-R u(2)-N(4)$ & $164.5(2)$ & $C(1)-R u(1)-N(1)$ & 157.5(1) & $C(2)-R u(2)-N(2)$ & $88.7(1)$ \\
\hline$\approx C(1)-R u(1)-N(3)$ & $95.6(1)$ & $\mathrm{Ru}(1)-\mathrm{C}(1)-\mathrm{O}(1)$ & $176.7(3)$ & $\mathrm{Ru}(1)-\mathrm{Ru}(2)-\mathrm{P}(1)$ & $172.3(1)$ & $\mathrm{Ru}(2)-\mathrm{C}(2)-\mathrm{O}(2)$ & $178.4(3)$ \\
\hline \multicolumn{8}{|c|}{ Bond Lengths for $\mathbf{1 2}$} \\
\hline$\cong R u(1)-R u(2)$ & $2.701(1)$ & $\mathrm{Ru}(2)-\mathrm{C}(25)$ & $2.009(6)$ & $\mathrm{Ru}(1)-\mathrm{C}(25)$ & $2.016(9)$ & $\mathrm{Ru}(2)-\mathrm{N}(6)$ & $2.093(5)$ \\
\hline $\mathrm{Ru}(1)-\mathrm{C}(24)$ & $2.009(6)$ & $\mathrm{Ru}(2)-\mathrm{N}(3)$ & $2.192(4)$ & $\mathrm{Ru}(1)-\mathrm{N}(2)$ & $2.194(7)$ & $\mathrm{Ru}(2)-\mathrm{N}(4)$ & $2.192(6)$ \\
\hline ฮี $R u(1)-N(1)$ & $2.196(5)$ & $C(26)-O(3)$ & $1.136(9)$ & $N(5)-N(6)$ & $1.365(8)$ & $C(27)-O(4)$ & $1.133(10)$ \\
\hline Z Ru(1)-N(5) & $2.097(5)$ & $\mathrm{C}(25)-\mathrm{O}(2)$ & $1.178(9)$ & $\mathrm{Ru}(2)-\mathrm{C}(24)$ & $2.033(8)$ & $\mathrm{C}(24)-\mathrm{O}(1)$ & $1.184(8)$ \\
\hline $\mathrm{Ru}(2)-\mathrm{C}(27)$ & $1.866(8)$ & $\mathrm{Ru}(1)-\mathrm{C}(26)$ & $1.868(7)$ & & & & \\
\hline \multicolumn{8}{|c|}{ Bond Angles for $\mathbf{1 2}$} \\
\hline $\mathrm{Ru}(1)-\mathrm{C}(24)-\mathrm{Ru}(2)$ & $83.9(3)$ & $N(1)-R u(1)-N(2)$ & $74.2(2)$ & $C(24)-R u(2)-C(25)$ & $92.9(3)$ & $N(3)-R u(2)-N(4)$ & $74.0(2)$ \\
\hline$C(24)-R u(1)-C(25)$ & $93.4(3)$ & $N(5)-R u(1)-C(26)$ & $174.5(3)$ & $\mathrm{C}(24)-\mathrm{Ru}(2)-\mathrm{N}(4)$ & $170.4(2)$ & $N(6)-R u(2)-C(27)$ & $174.6(3)$ \\
\hline $\mathrm{C}(24)-\mathrm{Ru}(1)-\mathrm{N}(1)$ & $169.4(3)$ & $\mathrm{Ru}(1)-\mathrm{C}(26)-\mathrm{O}(3)$ & $177.4(8)$ & $C(25)-R u(2)-N(3)$ & 167.9(3) & $\mathrm{Ru}(2)-\mathrm{C}(27)-\mathrm{O}(4)$ & 177.6(8) \\
\hline$C(25)-R u(1)-N(2)$ & $168.9(2)$ & $\mathrm{Ru}(1)-\mathrm{C}(25)-\mathrm{Ru}(2)$ & $84.3(3)$ & & & & \\
\hline \multicolumn{8}{|c|}{ Bond Lengths for 14A } \\
\hline $\mathrm{Ru}(1)-\mathrm{Ru}(2)$ & $2.6953(5)$ & $\mathrm{Ru}(2)-\mathrm{C}(6)$ & $2.016(4)$ & $\mathrm{Ru}(1)-\mathrm{C}(6)$ & $2.008(5)$ & $\mathrm{Ru}(2)-\mathrm{N}(4)$ & $2.132(4)$ \\
\hline $\mathrm{Ru}(1)-\mathrm{C}(5)$ & $2.024(4)$ & $\mathrm{Ru}(2)-\mathrm{N}(5)$ & $2.209(4)$ & $\mathrm{Ru}(1)-\mathrm{N}(2)$ & $2.197(4)$ & $\mathrm{Ru}(2)-\mathrm{N}(6)$ & $2.196(4)$ \\
\hline $\mathrm{Ru}(1)-\mathrm{N}(1)$ & $2.185(4)$ & $\mathrm{C}(1)-\mathrm{O}(1)$ & $1.136(6)$ & $N(3)-N(4)$ & $1.383(5)$ & $\mathrm{C}(2)-\mathrm{O}(2)$ & $1.149(6)$ \\
\hline $\mathrm{Ru}(1)-\mathrm{N}(3)$ & $2.124(4)$ & $\mathrm{C}(5)-O(5)$ & $1.177(5)$ & $\mathrm{Ru}(2)-C(5)$ & $2.017(4)$ & $\mathrm{C}(6)-O(6)$ & $1.182(5)$ \\
\hline $\mathrm{Ru}(2)-\mathrm{C}(2)$ & $1.862(5)$ & $\mathrm{Ru}(1)-\mathrm{C}(1)$ & $1.880(5)$ & & & & \\
\hline & & & Bond Angl & es for $14 A$ & & & \\
\hline $\mathrm{Ru}(1)-\mathrm{C}(5)-\mathrm{Ru}(2)$ & $\begin{array}{l}83.7(2) \\
946(2)\end{array}$ & $\begin{array}{l}N(1)-R u(1)-N(2) \\
N(3)-R u(1)-C(1)\end{array}$ & $\begin{array}{l}74.23(14) \\
1725(2)\end{array}$ & $\begin{array}{l}C(5)-R u(2)-C(6) \\
C(5)-R u(2)-N(5)\end{array}$ & $\begin{array}{r}94.6(2) \\
1693(2)\end{array}$ & $N(5)-R u(2)-N(6)$ & $\begin{array}{c}73.71(14) \\
1715(2)\end{array}$ \\
\hline $\begin{array}{l}C(5)-R U(1)-C(0) \\
C(5)-R u(1)-N(2)\end{array}$ & $170.6(2)$ & Ru(1)-C(1)-O(1) & $176.8(4)$ & $C(6)-R u(2)-N(6)$ & $168.6(2)$ & $\begin{array}{l}N(4)-R u(2)-C(2) \\
R u(2)-C(2)-O(2)\end{array}$ & $\begin{array}{l}1 / 1.5(2) \\
176.0(4)\end{array}$ \\
\hline $\mathrm{C}(6)-\mathrm{Ru}(1)-\mathrm{N}(1)$ & $167.6(2)$ & $\mathrm{Ru}(1)-\mathrm{C}(6)-\mathrm{Ru}(2)$ & $84.1(2)$ & & & & \\
\hline & & & Bond Len & is for 15 & & & \\
\hline $\mathrm{Ru}(1)-\mathrm{Ru}(2)$ & $2.685(1)$ & $\mathrm{Ru}(2)-\mathrm{N}(3)$ & $2.198(4)$ & $\mathrm{Ru}(1)-\mathrm{N}(6)$ & $2.103(4)$ & $\mathrm{Ru}(2)-\mathrm{N}(4)$ & $2.185(4)$ \\
\hline $\mathrm{Ru}(1)-\mathrm{N}(2)$ & $2.191(4)$ & $\mathrm{Ru}(2)-\mathrm{N}(5)$ & $2.141(5)$ & $\mathrm{Ru}(1)-\mathrm{C}(3)$ & $2.026(6)$ & $\mathrm{Ru}(2)-\mathrm{C}(2)$ & $1.851(7)$ \\
\hline $\mathrm{Ru}(1)-\mathrm{C}(1)$ & $1.873(6)$ & $\mathrm{Ru}(2)-\mathrm{C}(3)$ & $1.997(6)$ & $\mathrm{C}(1)-\mathrm{O}(1)$ & $1.134(8)$ & $\mathrm{Ru}(2)-\mathrm{C}(4)$ & $2.025(6)$ \\
\hline $\mathrm{Ru}(1)-\mathrm{C}(4)$ & $2.013(6)$ & $\mathrm{C}(2)-\mathrm{O}(2)$ & $1.144(9)$ & $\mathrm{C}(4)-\mathrm{O}(4)$ & $1.175(8)$ & $N(5)-N(6)$ & $1.393(6)$ \\
\hline $\mathrm{C}(3)-\mathrm{O}(3)$ & $1.188(8)$ & $\mathrm{Ru}(1)-\mathrm{N}(1)$ & $2.200(4)$ & & & & \\
\hline
\end{tabular}




\begin{tabular}{|c|c|c|c|c|c|c|c|}
\hline \multicolumn{8}{|c|}{ Bond Angles for $\mathbf{1 5}$} \\
\hline $\mathrm{Ru}(1)-\mathrm{C}(3)-\mathrm{Ru}(2)$ & $83.7(2)$ & $N(1)-R u(1)-N(2)$ & $75.6(2)$ & $C(3)-R u(2)-C(4)$ & $95.2(2)$ & $N(3)-R u(2)-N(4)$ & $75.0(2)$ \\
\hline$C(3)-R u(1)-C(4)$ & $94.7(2)$ & $N(6)-R u(1)-C(1)$ & $172.7(2)$ & $C(3)-R u(2)-N(4)$ & $169.4(2)$ & $N(5)-R u(2)-C(2)$ & $173.6(2)$ \\
\hline$C(3)-R u(1)-N(2)$ & $168.8(2)$ & $\mathrm{Ru}(1)-\mathrm{C}(1)-\mathrm{O}(1)$ & $176.8(6)$ & $\mathrm{C}(4)-\mathrm{Ru}(2)-\mathrm{N}(3)$ & 168.1(2) & $\mathrm{Ru}(2)-\mathrm{C}(2)-\mathrm{O}(2)$ & 178.5(6) \\
\hline$C(4)-R u(1)-N(1)$ & $171.1(2)$ & $\mathrm{Ru}(1)-\mathrm{C}(4)-\mathrm{Ru}(2)$ & $83.4(2)$ & & & & \\
\hline \multicolumn{8}{|c|}{ Bond Lengths for $\mathbf{1 6}$} \\
\hline $\mathrm{Ru}(1)-\mathrm{I}(1)$ & $2.731(2)$ & $\mathrm{Ru}(2)-\mathrm{N}(2)$ & $2.113(11)$ & $I(2)-I(3)$ & $2.926(2)$ & $\mathrm{Ru}(2)-\mathrm{N}(4)$ & $2.147(13)$ \\
\hline $\mathrm{Ru}(2)-I(1)$ & $2.733(1)$ & $\mathrm{Ru}(2)-\mathrm{C}(9)$ & $1.868(16)$ & $I(3)-I(4)$ & $2.902(2)$ & $\mathrm{Ru}(2)-\mathrm{C}(10)$ & $1.903(20)$ \\
\hline $\mathrm{Ru}(1)-\mathrm{P}(1)$ & $2.386(4)$ & $\mathrm{C}(7)-\mathrm{O}(1)$ & $1.131(19)$ & $\mathrm{Ru}(1)-\mathrm{N}(1)$ & $2.123(13)$ & $\mathrm{C}(8)-\mathrm{O}(2)$ & $1.153(25)$ \\
\hline $\mathrm{Ru}(1)-\mathrm{N}(3)$ & $2.105(10)$ & $C(9)-O(3)$ & $1.140(21)$ & $\mathrm{Ru}(1)-\mathrm{C}(7)$ & $1.877(15)$ & $C(10)-O(4)$ & $1.124(24)$ \\
\hline $\mathrm{Ru}(1)-\mathrm{C}(8)$ & $1.869(20)$ & $N(1)-N(2)$ & $1.360(16)$ & $\mathrm{Ru}(2)-\mathrm{P}(2)$ & $2.398(4)$ & $N(3)-N(4)$ & $1.347(16)$ \\
\hline \multicolumn{8}{|c|}{ Bond Angles for $\mathbf{1 6}$} \\
\hline $\mathrm{Ru}(1)-I(1)-\mathrm{Ru}(2)$ & 87.1(1) & $C(7)-R u(1)-C(8)$ & $90.8(7)$ & $I(1)-R u(1)-P(1)$ & 177.5(1) & $C(9)-R u(2)-C(10)$ & 89.6(8) \\
\hline$I(1)-R u(2)-P(2)$ & 178.9(1) & $\mathrm{Ru}(1)-\mathrm{C}(7)-\mathrm{O}(1)$ & 175.8(14) & $I(2)-I(3)-I(4)$ & 173.9(1) & $\mathrm{Ru}(1)-\mathrm{C}(8)-\mathrm{O}(2)$ & 173.9(14) \\
\hline$N(1)-R u(1)-N(3)$ & 88.2(4) & $\mathrm{Ru}(2)-\mathrm{C}(9)-\mathrm{O}(3)$ & 174.1(11) & $N(2)-R u(2)-N(4)$ & 88.7(5) & $\mathrm{Ru}(2)-\mathrm{C}(10)-\mathrm{O}(4)$ & $174.0(17)$ \\
\hline
\end{tabular}

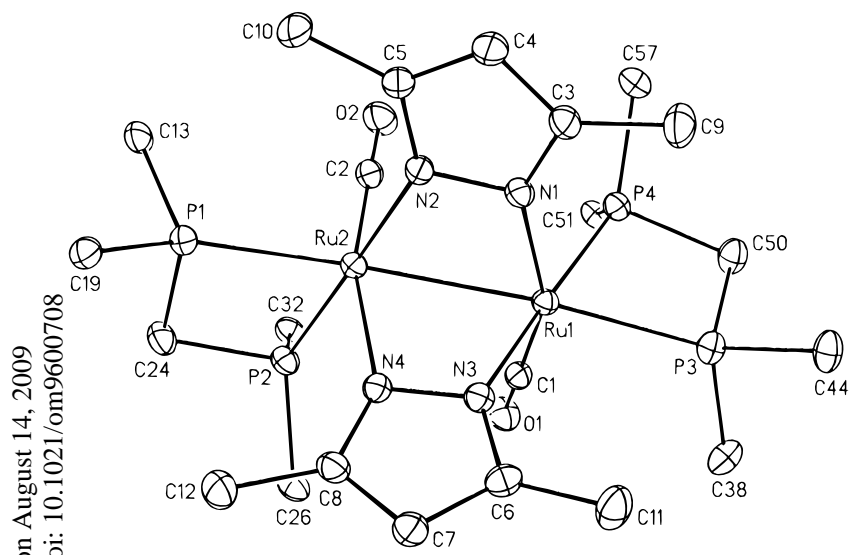

Figure 4. ORTEP plot of $\mathbf{1 1}$ with $50 \%$ probability elZ 1 ipsoids. Only the ipso-carbon atoms of the phenyl groups of dppm are shown for clarity.

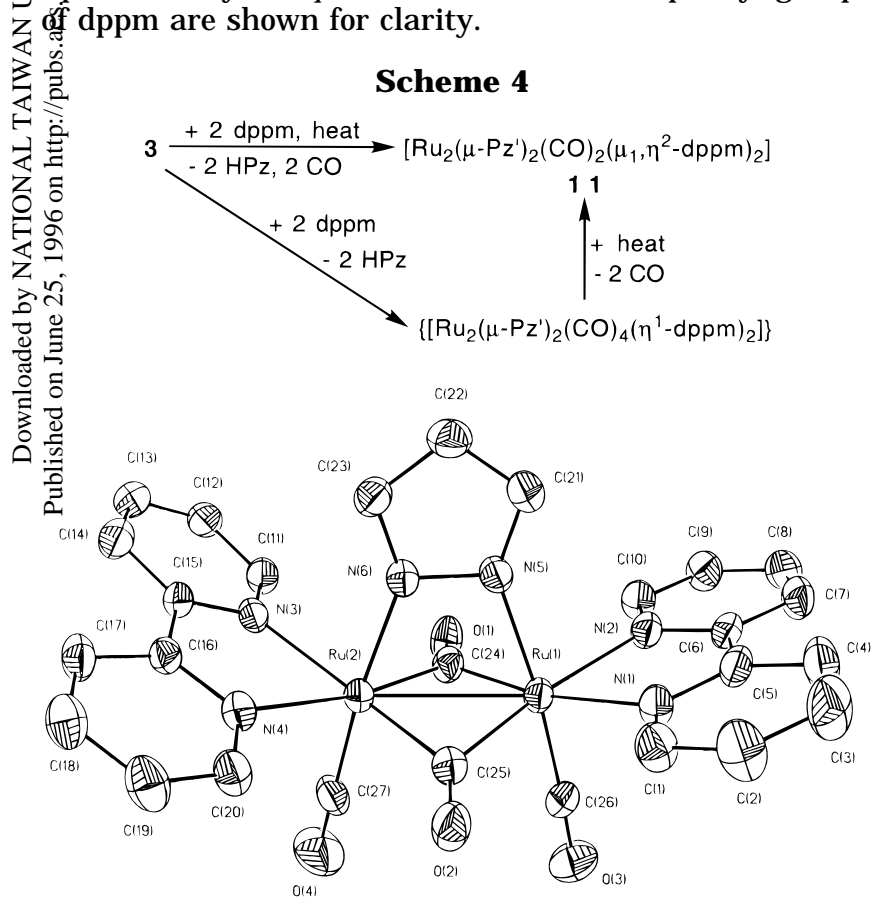

Figure 5. ORTEP plot of $[\mathbf{1 2}]^{+}$with $30 \%$ thermal ellipsoids.

Consistent with the structure of $\mathbf{1 0}$ (Figure 3b), phosphine groups can occupy the equatorial sites if necessary.

However, excepting the replacement of the axial $\mathrm{HL}$ groups, the less-flexible bidentate ligands such as bpy and phen can cleave one $\mu-\mathrm{O}_{2} \mathrm{CMe}$ or $\mu$-L bridge, but without losing any carbonyl in $\mathbf{1 - 3}$, to afford $\left[\mathrm{Ru}_{2}(\mu-\right.$

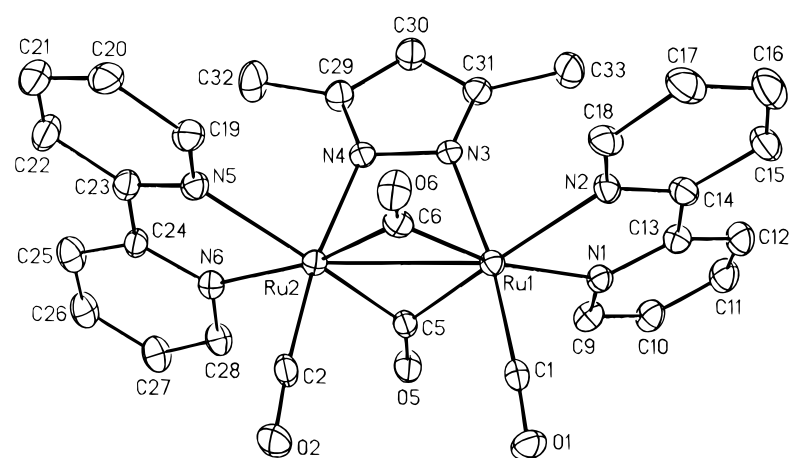

Figure 6. ORTEP plot of $[\mathbf{1 4 A}]^{+}$with $50 \%$ probability ellipsoids.

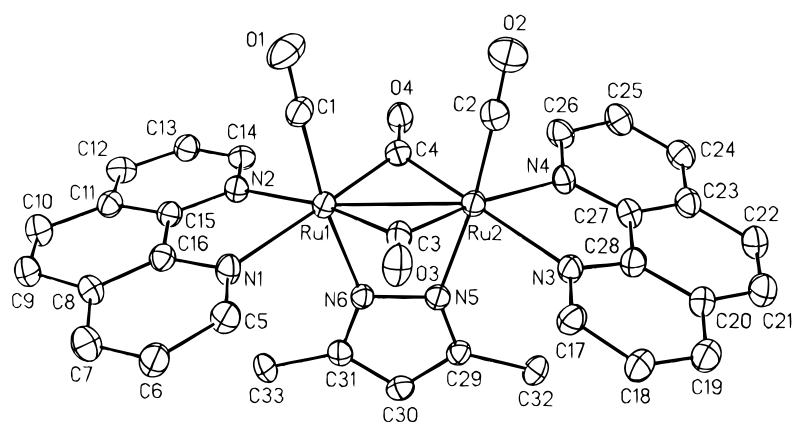

Figure 7. ORTEP plot of $[\mathbf{1 5}]^{+}$with $50 \%$ probability ellipsoids.

$$
\begin{aligned}
& \text { Scheme } 5 \\
& 1 \stackrel{+2 \mathrm{~N}-\mathrm{N}, \mathrm{NaBPh}_{4}}{-2 \mathrm{HPz}, \mathrm{Pz}}-\left[\mathrm{Ru}_{2}(\mu-\mathrm{Pz})(\mu-\mathrm{CO})_{2}(\mathrm{CO})_{2}\left(\mu_{1}, \eta^{2}-(\mathrm{N}-\mathrm{N})\right)_{2}\right]\left[\mathrm{BPh}_{4}\right] \\
& \mathrm{N}-\mathrm{N}=\text { bpy (12), phen (13) } \\
& \begin{array}{c}
2 \underset{-2 \mathrm{HPz}^{\prime}, \mathrm{MeCO}_{2}^{-}}{\stackrel{+}{-}}\left[\mathrm{Ru}_{2}\left(\mu-\mathrm{Pz}^{\prime}\right)(\mu-\mathrm{CO})_{2}(\mathrm{CO})_{2}\left(\mu_{1}, \eta^{2}-(\mathrm{N}-\mathrm{N})\right)_{2}\right]\left[\mathrm{PF}_{6}\right] \\
\mathrm{N}-\mathrm{N}=\text { bpy (14), phen (15) }
\end{array} \\
& 3 \underset{-2 \mathrm{~N}-\mathrm{N}, \mathrm{NaPF}_{6}}{-2 \mathrm{HPz}^{\prime}, \mathrm{Pz}^{\prime-}}\left[\mathrm{Ru}_{2}(\mu-\mathrm{Pz})(\mu-\mathrm{CO})_{2}(\mathrm{CO})_{2}\left(\mu_{1}, \eta^{2}-(\mathrm{N}-\mathrm{N})\right)_{2}\right]\left[\mathrm{PF}_{6}\right]
\end{aligned}
$$

$\left.\mathrm{L})(\mu-\mathrm{CO})_{2}(\mathrm{CO})_{2}\left(\mu_{1}, \eta^{2}-(\mathrm{N}-\mathrm{N})\right)_{2}\right]^{+}(\mathrm{L}=\mathrm{Pz}, \mathrm{N}-\mathrm{N}=$ bpy $\left([\mathbf{1 2}]^{+}\right)$, phen $\left([\mathbf{1 3}]^{+}\right) ; \mathrm{L}=\mathrm{Pz}^{\prime}, \mathrm{N}-\mathrm{N}=$ bpy $\left([\mathbf{1 4}]^{+}\right)$, phen $\left([15]^{+}\right)$) (Scheme 5). The crystal structures of 12, 14, and $\mathbf{1 5}$ were carried out to confirm the formulation, and it was found that in each asymmetric unit of the crystal used were two similar diruthenium molecules, 14A and 14B, found for 14, although only one dimer was observed for 12 and $\mathbf{1 5}$. The structures of $[\mathbf{1 2}]^{+},[\mathbf{1 4 A}]^{+}$, and $\left[\mathbf{1 5}^{+}\right.$are shown in Figures 5-7, respectively. Apparently the more severe steric effect required by bpy 


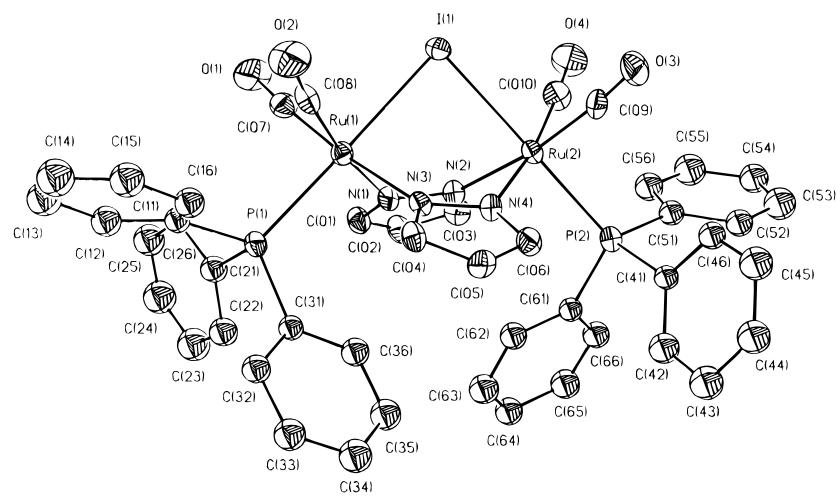

Figure 8. ORTEP plot of $[\mathbf{1 6}]^{+}$with $50 \%$ probability ellipsoids.

and phen, relative to dppm, ${ }^{5,6}$ results in a geometry in 12-15 totally different from that in $\mathbf{1 1}$.

The $\mu-\mathrm{O}_{2} \mathrm{CMe}$ linkage was previously reported to be cleavable, and the product $\left[\mathrm{Ru}_{2}\left(u-\mathrm{O}_{2} \mathrm{CMe}\right)(\mu-\mathrm{CO})_{2}(\mathrm{CO})_{2^{-}}\right.$ $\left.\left(\mu_{1}, \eta^{2}-(\mathrm{N}-\mathrm{N})\right)_{2}\right]^{+}$can be obtained from the reaction of catena-[Ru $\left.\left(\mathrm{O}_{2} \mathrm{CMe}\right)(\mathrm{CO})_{2}\right]$ with bpy or phen in $\mathrm{EtOH} .^{7}$ Here we wish to add that the apparently strong $\mu-\mathrm{L}$ Enkage, previously reported to be a stable bridge in all dirrhodium and -iridium complexes, ${ }^{1}$ can be cleaved. The coeaction between the heterobridged dimer 2 and $\mathrm{N}-\mathrm{N}$ \pm fid not give a mixture of $\left[\mathrm{Ru}_{2}\left(\mu-\mathrm{O}_{2} \mathrm{CMe}\right)(\mu-\mathrm{CO})_{2}(\mathrm{CO})_{2^{-}}\right.$ $\left.\left.u_{1}, \eta^{2}-(\mathrm{N}-\mathrm{N})\right)_{2}\right]^{+}$and $\left[\mathrm{Ru}_{2}(\mu-\mathrm{L})(\mu-\mathrm{CO})_{2}(\mathrm{CO})_{2}\left(\mu_{1}, \eta^{2}-(\mathrm{N}-\right.\right.$ $\left.\mathrm{E}_{0}()_{2}\right]^{+}$but only the latter one, consistent with the weaker $\mu$-acetato linkage compared with the $\mu$-Pz' $\geq$ Inkage, and the cl eaving process is also sel ective when 夠y or phen is used.

$\mathrm{z}$ The comparable Ru-Ru distances of 2.701(1) $\AA$ in $[22]^{+}, 2.6953(5) \AA$ in $[\mathbf{1 4 A}]^{+}, 2.6988(5) \AA$ in $[\mathbf{1 4 B}]^{+}$, 685(1) $\AA$ in $[15]^{+}, 2.701(1) \AA$ in $\left[\mathrm{Ru}_{2}\left(\mu-\mathrm{O}_{2} \mathrm{CMe}\right)(\mu-\mathrm{CO})_{2}^{-}\right.$ $\left.(\mathrm{EO})_{2}\left(\mu_{1}, \eta^{2}-\mathrm{bpy}\right)_{2}\right]^{+}$, 7a and 2.709(1) $\AA$ in $\left[\mathrm{Ru}_{2}\left(\mu-\mathrm{O}_{2} \mathrm{CM} \mathrm{e}\right)-\right.$

$\left.\left.{ }_{\mathrm{G}}-\mathrm{CO}\right)_{2}(\mathrm{CO})_{2}\left(\mu_{1}, \eta^{2} \text {-phen }\right)_{2}\right]^{+} 7 \mathrm{~b}$ can be attributed to the silmilar flexibility of two bidentate ligands bpy and phen with the averaged torsion angles $\angle \mathrm{N}-\mathrm{C}-\mathrm{C}-\mathrm{N}$, such as \% $\mathrm{N}(3)-\mathrm{C}(15)-\mathrm{C}(16)-\mathrm{N}(4)$ in 12 , less than $6.4^{\circ}$. The ientical distance of 2.701(1) $\AA$ found in both cations $2^{+}$and $\left[\mathrm{Ru}_{2}\left(u-\mathrm{O}_{2} \mathrm{CMe}\right)(\mu-\mathrm{CO})_{2}(\mathrm{CO})_{2}\left(\mu_{1}, \eta^{2}-\mathrm{bpy}\right)_{2}\right]^{+}$probâbly indicates $\mathrm{a} \mu-\mathrm{O}_{2} \mathrm{CMe}$ linkage in a steric bulk similar t a $\mu$-Pz linkage and further supports the facile geplacement of two $\mu-\mathrm{O}_{2} \mathrm{CMe}$ linkages in $\mathbf{1}$ by $\mu-\mathrm{Pz}$ groups to give 4 (Scheme 1).

Reactions with Electrophiles. Electrophiles such as $I_{2}$ can react with 4 . However, it was strange to find that the reaction should consume up to 2 equiv of $I_{2}$ to complete the reaction for every dinuclear compound 4. Later, the crystal structure of the product compound was revealed to have an $\mathrm{I}_{3}^{-}$as a counterion for $\left[\mathrm{Ru}_{2}(\mu-\right.$ $\left.\mathrm{Pz})_{2}(\mu-\mathrm{I})(\mathrm{CO})_{4}\left(\mathrm{PPh}_{3}\right)_{2}\right]^{+}\left([\mathbf{1 6}]^{+}\right.$; cf., Figure 8 and Scheme 6). Though with two $\mu$-Pz linkages, the two Ru atoms are separated by $3.762(2) \AA$, indicating no metal-metal bonding interactions. Obviously, the electrophilic reagent $I_{2}$ can cleave the $\mathrm{Ru}-\mathrm{Ru}$ bond and raise the oxidation state of each Ru atom, but cannot break the $\mu$-azolato linkage. Such a feature was previously ob-

(6) (a) Puddephatt, R. J . Chem. Soc. Rev. 1983, 12, 99. (b) Balch, A L. Homogeneous Catalysis with Metal Phosphine Complexes; Pignolet, L. H., Ed.; Plenum: New York, 1983; pp 167-213. (c) Chaudret, B.; Delavaux, B.; Poilblanc, R. Coord. Chem. Rev. 1988, 86, 191.

(7) (a) Steyn, M. M. d. V.; Singleton, E. Acta Crystallogr. 1988, C44, 1722. (b) Frediani, P.; Bianchi, M.; Salvini, A.; Guarducci, R.; Carluccio, L. C.; Piacenti, F.; I anelli, S.; Nardelli, M. J . Organomet. Chem. 1993, $463,187$.

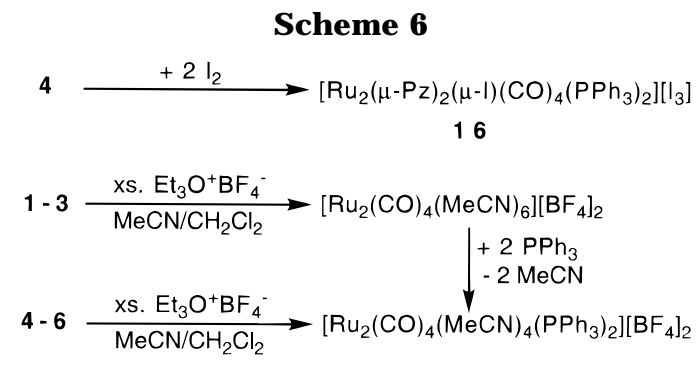

served in the dirhodium and -iridium systems ${ }^{1}$ but not yet reported in the diruthenium system. ${ }^{2}$ From the crystal structures of $2,5,10-12$, and $14-16$, it is apparent that the $\mu$-azolato linkage is flexible to maintain two metal fragments in close proximity, both within and beyond the requirements of metal-metal interactions in the dimetal system.

From the aforementioned results, one or two $\mu$-azolato linkages in 1-3 appear to be able to survive during a nucleophilic or electrophilic reaction (Schemes 1-5). However, some other reactions are present enabling removal of all the $\mu$-acetato and -azolato linkages in the compounds.

An electrophilic reagent such as $\mathrm{Et}_{3} \mathrm{O}^{+} \mathrm{BF}_{4}^{-}$in the presence of $\mathrm{MeCN}$ was previously reported by our laboratory to convert a series of $\left[\mathrm{Ru}_{2}(\mathrm{CO})_{4}\left(\mu-\mathrm{O}_{2} \mathrm{CMe}\right)_{2^{-}}\right.$ $\left.\left(L^{\prime}\right)_{2}\right]\left(L^{\prime}=\mathrm{MeCN}\right.$ or phosphine $\left.\left(\mathrm{PR}_{3}\right)\right)$ into $\left[\mathrm{Ru}_{2}(\mathrm{CO})_{4^{-}}\right.$ $\left.(\mathrm{MeCN})_{6}\right]\left[\mathrm{BF}_{4}\right]_{2}$ and $\left[\mathrm{Ru}_{2}(\mathrm{CO})_{4}(\mathrm{MeCN})_{4}\left(\mathrm{PR}_{3}\right)_{2}\right]\left[\mathrm{BF}_{4}\right]_{2}$, probably involving an abstraction reaction by transformation of a $\mu$-acetato bridge into the weakly coordinating ethyl acetate. ${ }^{4 \mathrm{~b}}$ We found that a similar reaction also occurs for 1-3 and 4-6 into $\left[\mathrm{Ru}_{2}(\mathrm{CO})_{4}(\mathrm{MeCN})_{6}\right]-$ $\left[\mathrm{BF}_{4}\right]_{2}$ and $\left[\mathrm{Ru}_{2}(\mathrm{CO})_{4}(\mathrm{MeCN})_{4}\left(\mathrm{PPh}_{3}\right)_{2}\right]\left[\mathrm{BF}_{4}\right]_{2}$, respectively. The former product, $\left[\mathrm{Ru}_{2}(\mathrm{CO})_{4}(\mathrm{MeCN})_{6}\right]\left[\mathrm{BF}_{4}\right]_{2}$, can be converted by adding $\mathrm{PPh}_{3}$ into the latter one, $\left[\mathrm{Ru}_{2}(\mathrm{CO})_{4}(\mathrm{MeCN})_{4}\left(\mathrm{PPh}_{3}\right)_{2}\right]\left[\mathrm{BF}_{4}\right]_{2}$ (Scheme 6). ${ }^{4 a}$

\section{Conclusion}

Our investigation into the diruthenium carbonyl complexes containing the $\mu$-azol ato linkage resulted in the specific synthesis of $\mathbf{1 - 3}$ in satisfactory yield. Both nucleophilic and electrophilic reactions of $\mathbf{1}-\mathbf{3}$ produce selectively one type of product containing either heteroor homobridges (Schemes 1-6) as confirmed in eight $\mathrm{X}$-ray crystal structures (Figures 1-8). The $\mu$-azolato linkage has been demonstrated to promote formation of novel compounds such as $\mathbf{1 1}$ and to maintain two ruthenium fragments in close proximity, both within and beyond the requirements of metal-metal interactions. More importantly, if necessary, the $\mu$-azolato linkages can be cleaved partially by using the lessflexible bidentate nucleophiles such as bpy or phen or removed totally by using el ectrophiles such as trialkyloxonium reagents. The latter reaction can produce potentially versatile compounds such as $\left[\mathrm{Ru}_{2}(\mathrm{CO})_{4^{-}}\right.$ $\left.(\mathrm{MeCN})_{6}\right]\left[\mathrm{BF}_{4}\right]_{2}$ and $\left[\mathrm{Ru}_{2}(\mathrm{CO})_{4}(\mathrm{MeCN})_{4}\left(\mathrm{PPh}_{3}\right)_{2}\right]\left[\mathrm{BF}_{4}\right]_{2} \cdot{ }^{4}$

\section{Experimental Section}

General Comments. All solvents were dried and purified by standard methods [ethers, paraffins, and arenes from potassium with benzophenone as indicator; halocarbons and acetonitrile from $\mathrm{CaH}_{2}$; al cohols from the corresponding alkoxide] and were freshly distilled under nitrogen immediately before use. All reactions and manipulations were carried out in standard Schlenk ware, connected to a switchable double 
manifold providing vacuum and nitrogen. Reagents and phosphines were used as supplied by either Aldrich or Strem. ${ }^{1} \mathrm{H}$ and ${ }^{31} \mathrm{P}$ NMR spectra were measured on a Bruker AM-200 $\left({ }^{1} \mathrm{H}, 200 \mathrm{MHz}\right)$, Bruker AMC-400, or Varian Unity Plus-400 $\left({ }^{1} \mathrm{H}, 400 \mathrm{M} \mathrm{Hz}\right.$; $\left.{ }^{31} \mathrm{P}, 162 \mathrm{MHz}\right) \mathrm{NMR}$ spectrometer. ${ }^{1 \mathrm{H}}$ chemical shifts ( $\delta$ in ppm, $J$ in $\mathrm{Hz}$ ) are defined as positive downfield relative to internal $\mathrm{MeSi}_{4}$ (TMS) or the deuterated solvent, while ${ }^{31} \mathrm{P}$ chemical shifts are defined as positive downfield relative to external $85 \% \mathrm{H}_{3} \mathrm{PO}_{4}$. The IR spectra were recorded on a Hitachi Model 270-30 or Bio-Rad FTS 175 instrument. The following abbreviations were used: $s$, strong; $m$, medium; w, weak; s, singlet; d, doublet; t, triplet; dd, doublet of doublet; br, broad unresolved signal. Microanalyses were carried out by the staff of the Microanalytical Service of the Department of Chemistry, National Cheng Kung University.

Synthesis of $\left[\mathbf{R u}_{\mathbf{2}}(\mathbf{C O})_{\mathbf{4}}(\boldsymbol{\mu}-\mathbf{P z})_{\mathbf{2}}(\mathbf{H P z})_{2}\right](\mathbf{1})$. In a $100-\mathrm{mL}$ Schlenk flask was added catena- $\left[\mathrm{Ru}\left(\mathrm{O}_{2} \mathrm{CMe}\right)(\mathrm{CO})_{2}\right](1.0 \mathrm{~g}, 2.33$ $\mathrm{mmol}), \mathrm{HPz}(0.83 \mathrm{~g}, 12.2 \mathrm{mmol}), 5 \mathrm{~mL}$ of $\mathrm{Et}_{3} \mathrm{~N}$, and $30 \mathrm{~mL}$ of $\mathrm{EtOH}$ at room temperature. The mixture was then heated under reflux for $2 \mathrm{~h}$ and cooled to room temperature. The solvent and $\mathrm{Et}_{3} \mathrm{~N}$ were removed under vacuum and the resulting solid residue was redissolved in $5 \mathrm{~mL}$ of $\mathrm{MeOH}$. U pon addition of $50 \mathrm{~mL}$ of $\mathrm{H}_{2} \mathrm{O}$, a milky yellow precipitate formed immediately, which was collected on a medium frit. Recrystallization from $\mathrm{CH}_{2} \mathrm{Cl}_{2} / \mathrm{MeOH}$ afforded the pure product in $1 \%$ yield. Alternatively, as described below for 3, 1 can also prepared from $\left[\mathrm{Ru}_{2}(\mathrm{CO})_{4}(\mathrm{MeCN})_{6}\right]\left[\mathrm{BF}_{4}\right]_{2}$ and $\mathrm{HPz} / \mathrm{Et}_{3} \mathrm{~N}$. .nal. Calcd for $\mathrm{C}_{16} \mathrm{H}_{14} \mathrm{~N}_{8} \mathrm{O}_{4} \mathrm{Ru}_{2}$ : C, 32.88; $\mathrm{H}, 2.41 ; \mathrm{N}, 19.17$. Eound: C, 32.54; $\mathrm{H}, 2.39 ; \mathrm{N}, 18.85$. ${ }^{1} \mathrm{H} N \mathrm{NMR}\left(25^{\circ} \mathrm{C}, 400 \mathrm{MHz}\right.$, acetone- $\left.\mathrm{d}_{6}\right): \mathrm{NH}$ at $12.87(\mathrm{br}, 2 \mathrm{H}) ; \mathrm{H}^{3}$ or $\mathrm{H}^{5}$ on $\mathrm{HPz}$ at 8.10 (m, $2 \mathrm{H}), 7.83(\mathrm{~m}, 2 \mathrm{H}) ; \mathrm{H}^{3}$ and $\mathrm{H}^{5}$ on $\mu-\mathrm{Pz}$ at $7.09(\mathrm{~m}, 4 \mathrm{H}) ; \mathrm{H}^{4}$ 年 $\mathrm{HPZ}$ at $6.64(\mathrm{~m}, 2 \mathrm{H},) ; \mathrm{H}^{4}$ on $\mu$-Pz at $6.02(\mathrm{~m}, 2 \mathrm{H})$. IR: o $2016 \mathrm{~s}, 1964 \mathrm{~m}, 1934 \mathrm{~s} \mathrm{~cm}^{-1}$ in $\mathrm{KBr}$.

Synthesis of $\left[R u_{2}(C O)_{4}\left(\mu-P^{\prime}\right)\left(\mu-O_{2} C M e\right)\left(H P z^{\prime}\right)_{2}\right]$ $\mathrm{Z}$ the yellow compound $\mathbf{2}$ was prepared from catena-[Ru( $\mathrm{O}_{2}$ (E) $(\mathrm{CO})_{2}$ ] using $\mathrm{HPz}^{\prime}$ in a procedure similar to that used for Theyield is 87\%. Anal. Calcd for $\mathrm{C}_{21} \mathrm{H}_{16} \mathrm{~N}_{6} \mathrm{O}_{6} \mathrm{Ru}_{2}$ : C, 38.18; iq 3.97; N, 12.72. Found: C, 38.51; H, 3.99; N, 12.39. ${ }^{1} \mathrm{H}$ NMR $\left(25^{\circ} \mathrm{C}, 200 \mathrm{MHz}, \mathrm{CDCl}_{3}\right): \mathrm{NH}$ at $10.92(\mathrm{br}, 2 \mathrm{H}) ; \mathrm{H}^{4}$ on $\mathrm{HPz}^{\prime}$ at $6.02(\mathrm{~s}, 1 \mathrm{H}), 6.01(\mathrm{~s}, 1 \mathrm{H}) ; \mathrm{H}^{4}$ on $\mu-\mathrm{Pz}^{\prime}$ at $5.57(\mathrm{~s}, 1 \mathrm{H}) ; \mathrm{Me}^{3}$ बे $\mathrm{Me}^{5}$ on $\mathrm{HPz}^{\prime}$ at $2.48(\mathrm{br}, 6 \mathrm{H}), 2.29(\mathrm{br}, 6 \mathrm{H}) ; \mathrm{Me}^{3}$ or $\mathrm{Me}^{5}$ on $\mu \mathrm{Pz}^{\prime}$ at $1.38(\mathrm{br}, 6 \mathrm{H}) ; \mu-\mathrm{O}_{2} \mathrm{CMe}$ at $2.15(\mathrm{~s}, 3 \mathrm{H})$. IR: $\mathrm{V}_{\mathrm{CO}}$, $2024 \mathrm{~s}, 1974 \mathrm{~m}, 1940 \mathrm{~s} \mathrm{~cm}^{-1}$ in $\mathrm{CH}_{2} \mathrm{Cl}_{2} ; \mathrm{v}_{\mathrm{NH}}, 3328 \mathrm{w}, \mathrm{v}_{\mathrm{CO}}, 2024$ s. $1970 \mathrm{~m}, 1942 \mathrm{~s} \mathrm{~cm}^{-1}$ in KBr.

E Synthesis of $\left[\mathrm{Ru}_{\mathbf{2}}(\mathrm{CO})_{4}\left(\mu-\mathrm{Pz}^{\prime}\right)_{2}\left(\mathrm{HPz}^{\prime}\right)_{2}\right]$ (3). To the solu世्won of $\left[\mathrm{Ru}_{2}(\mathrm{CO})_{4}(\mathrm{MeCN})_{6}\right]\left[\mathrm{BF}_{4}\right]_{2}$, prepared in situ by the eaction of $\left[\mathrm{Ru}_{2}(\mathrm{CO})_{4}\left(\mu-\mathrm{O}_{2} \mathrm{CMe}\right)_{2}(\mathrm{MeCN})_{2}\right](0.28 \mathrm{mmol})$ with Excess $\mathrm{Et}_{3} \mathrm{O}^{+} \mathrm{BF}_{4}{ }^{-}, 4$ in a $100-\mathrm{mL}$ Schlenk flask was added $\mathrm{HPz}^{\prime}$ $(0.14 \mathrm{~g}, 1.4 \mathrm{mmol}), 1 \mathrm{~mL}$ of $\mathrm{Et}_{3} \mathrm{~N}$, and $20 \mathrm{~mL}$ of $\mathrm{MeOH}$ at room temperature. The mixture was then heated under reflux for $2 \mathrm{~h}$ and cooled to room temperature. The volume of the solution was reduced to $\mathrm{ca} .5 \mathrm{~mL}$ under vacuum. Upon addition of $40 \mathrm{~mL}$ of $\mathrm{H}_{2} \mathrm{O}$, a milky yellow precipitate formed immediately, which was collected on a medium frit. Recrystallization from $\mathrm{CH}_{2} \mathrm{Cl}_{2} / \mathrm{MeOH}$ afforded the pure product in 83\% yield. Anal. Calcd for $\mathrm{C}_{24} \mathrm{H}_{30} \mathrm{~N}_{8} \mathrm{O}_{4} \mathrm{Ru}_{2}$ : C, 41.38; $\mathrm{H}, 4.34$; $\mathrm{N}, 16.08$. Found: $\mathrm{C}, 41.35 ; \mathrm{H}, 4.28 ; \mathrm{N}, 15.83$. ${ }^{1} \mathrm{H}$ NMR (25 $\left.{ }^{\circ} \mathrm{C}, 200 \mathrm{MHz}, \mathrm{CDCl}_{3}\right): \mathrm{NH}$ at $9.80(\mathrm{br}, 2 \mathrm{H}) ; \mathrm{H}^{4}$ on $\mathrm{HPz}^{\prime}$ at $6.05(\mathrm{~s}, 1 \mathrm{H}), 5.81(\mathrm{~s}, 1 \mathrm{H}) ; \mathrm{H}^{4}$ on $\mu-\mathrm{Pz}^{\prime}$ at $5.31(\mathrm{~s}, 1 \mathrm{H}) ; \mathrm{Me}^{3}$ or $\mathrm{Me}^{5}$ on $\mathrm{HPz}^{\prime}$ at $2.55(\mathrm{br}, 6 \mathrm{H}), 2.24(\mathrm{br}, 6 \mathrm{H}) ; \mathrm{Me}^{3}$ or $\mathrm{Me}^{5}$ on $\mu$-Pz at $1.55(\mathrm{br}, 12 \mathrm{H})$. IR: $\mathrm{V}_{\mathrm{CO}}, 2016 \mathrm{~s}, 1968 \mathrm{~m}, 1934 \mathrm{~s} \mathrm{~cm}^{-1}$ in $\mathrm{CH}_{2} \mathrm{Cl}_{2} ; \mathrm{v}_{\mathrm{NH}}, 3424 \mathrm{w}, \mathrm{v}_{\mathrm{CO}}, 2012 \mathrm{~s}, 1960 \mathrm{~m}, 1928 \mathrm{~s} \mathrm{~cm}^{-1}$ in $\mathrm{KBr}$.

Reaction between $\mathbf{1}$ and $\mathbf{P P h}_{\mathbf{3}}$. In a 100-mL Schlenk flask was added $1(0.100 \mathrm{~g}, 0.17 \mathrm{mmol}), \mathrm{PPh}_{3}(0.18 \mathrm{~g}, 0.69$ $\mathrm{mmol}$ ), and $25 \mathrm{~mL}$ of $\mathrm{MeCN}$ at room temperature. The solution gradually formed pale yellow precipitate. After 0.5 $h$, the yellow solid was collected on a medium frit, washed three times with $5 \mathrm{~mL}$ of $\mathrm{MeCN}$, and dried in vacuo to give $0.096 \mathrm{~g}(80 \%)$. This solid was identified as $\left[\mathrm{Ru}_{2}(\mathrm{CO})_{4}(u-\mathrm{Pz})_{2^{-}}\right.$
$\left(\mathrm{PPh}_{3}\right)_{2}$ ] by comparison of both the NMR and IR spectral evidences with those reported.2c

Reaction between $\mathbf{2}$ and $\mathbf{P P h}$. The yellow compound $\mathbf{5}$ was prepared from $\mathbf{2}$ in a procedure similar to that used for $\mathbf{4}$. The yield is 97\%. Anal. Calcd for $\mathrm{C}_{47} \mathrm{H}_{40} \mathrm{~N}_{2} \mathrm{O}_{6} \mathrm{P}_{2} \mathrm{Ru}_{2}$ : C, 56.85; $\mathrm{H}, 4.06$; N, 2.82. Found: C, 56.78; $\mathrm{H}, 4.04 ; \mathrm{N}, 2.72$. ${ }^{1} \mathrm{H} N M R$ $\left(25^{\circ} \mathrm{C}, 200 \mathrm{MHz}, \mathrm{CDCl}_{3}\right): \mathrm{PPh}_{3}$ at $7.60(\mathrm{br}, 12 \mathrm{H}), 7.40(\mathrm{~m}, 18$ $\mathrm{H}) ; \mathrm{H}^{4}$ on $\mu-\mathrm{Pz}^{\prime}$ at $5.67(\mathrm{~s}, 1 \mathrm{H}) ; \mathrm{Me}^{3}$ or $\mathrm{Me}^{5}$ on $\mu-\mathrm{Pz}^{\prime}$ at 1.57 (br, $6 \mathrm{H}) ; \mu-\mathrm{O}_{2} \mathrm{CMe}$ at $1.75(\mathrm{~s}, 3 \mathrm{H}) .{ }^{31} \mathrm{P}\left\{{ }^{1} \mathrm{H}\right\} \mathrm{NMR}\left(25^{\circ} \mathrm{C}, 162\right.$ $\mathrm{MHz}_{\mathrm{CDCl}}$ ): 12.50 (s, $\left.2 \mathrm{P}\right)$. IR $\left(\mathrm{CH}_{2} \mathrm{Cl}_{2}\right)$ : v $\mathrm{v}_{\mathrm{CO}}, 2028 \mathrm{~s}, 1982$ $\mathrm{m}, 1954 \mathrm{~s} \mathrm{~cm}^{-1}$.

Reaction between $\mathbf{3}$ and $\mathbf{P P h}$. The yellow compound $\mathbf{6}$ was prepared from $\mathbf{3}$ in a procedure similar to that used for $\mathbf{4}$. The yield is 94\%. Anal. Calcd for $\mathrm{C}_{50} \mathrm{H}_{44} \mathrm{~N}_{4} \mathrm{O}_{4} \mathrm{P}_{2} \mathrm{Ru}_{2}$ : C, 58.36; $\mathrm{H}, 4.31 ; \mathrm{N}, 5.44$. Found: $\mathrm{C}, 58.29 ; \mathrm{H}, 4.28 ; \mathrm{N}, 5.57$. ${ }^{1} \mathrm{H} N M R$ $\left(25,200 \mathrm{MHz}, \mathrm{CDCl}_{3}\right): \mathrm{PPh}_{3}$ at $7.54-7.23(\mathrm{~m}, 30 \mathrm{H}) ; \mathrm{H}^{4}$ on $\mu-\mathrm{Pz}^{\prime}$ at $5.63(\mathrm{~s}, 1 \mathrm{H}), 5.58(\mathrm{~s}, 1 \mathrm{H}) ; \mathrm{Me}^{3}$ or $\mathrm{Me}^{5}$ on $\mu-\mathrm{Pz}^{\prime}$ at 2.27 $(\mathrm{s}, 3 \mathrm{H}), 1.80(\mathrm{~s}, 3 \mathrm{H}), 1.51(\mathrm{~s}, 3 \mathrm{H}), 1.46(\mathrm{~s}, 3 \mathrm{H}) .{ }^{31 P}\left\{{ }^{1} \mathrm{H}\right\}$ NMR $\left(25^{\circ} \mathrm{C}, 162 \mathrm{MHz} \mathrm{CDCl}_{3}\right): 12.69(\mathrm{~s}, 2 \mathrm{P})$. IR $\left(\mathrm{CH}_{2} \mathrm{Cl}_{2}\right)$ : $\mathrm{V}_{\mathrm{CO}}, 2016 \mathrm{~s}, 1990 \mathrm{~m}, 1944 \mathrm{~s}, 1924 \mathrm{~m} \mathrm{~cm}^{-1}$.

Reaction between 1 and dppm. The yellow compound $\mathbf{7}$ was prepared from $\mathbf{1}$ using dppm in a procedure similar to that used for 4. The yield is $93 \%$. Anal. Calcd for $\mathrm{C}_{60} \mathrm{H}_{50}$ $\mathrm{N}_{4} \mathrm{O}_{4} \mathrm{P}_{4} \mathrm{Ru}_{2}$ : C, 59.22; $\mathrm{H}, 4.14 ; \mathrm{N}, 4.60$. Found: $\mathrm{C}, 59.28 ; \mathrm{H}$, 4.08; N, 4.59. ${ }^{1} \mathrm{H} N M R\left(25^{\circ} \mathrm{C}, 200 \mathrm{MHz}, \mathrm{CDCl}_{3}\right): \mathrm{PPh}_{3}$ at 7.65-7.10 (m, $40 \mathrm{H}) ; \mathrm{H}^{3}$ and $\mathrm{H}^{5}$ on $\mu-\mathrm{Pz}$ at $6.46(\mathrm{~d}, 4 \mathrm{H}, \mathrm{J}=$ 2.2); $\mathrm{H}^{4}$ on $\mu$-Pz at $5.63(\mathrm{t}, 2 \mathrm{H}) ; \mathrm{CH}_{2}$ of dppm at $3.47(\mathrm{~m}, 4 \mathrm{H})$. ${ }^{31} \mathrm{P}\left\{{ }^{1} \mathrm{H}\right\}$ NMR $\left(25^{\circ} \mathrm{C}, 162 \mathrm{MHz} \mathrm{CDCl}_{3}\right): 12.12(\mathrm{~m}, 2 \mathrm{P}),-26.18$ (m, $2 \mathrm{P})$. IR $\left(\mathrm{CH}_{2} \mathrm{Cl}_{2}\right): \mathrm{V}_{\mathrm{CO}}, 2024 \mathrm{~s}, 1982 \mathrm{~m}, 1954 \mathrm{~s} \mathrm{~cm}^{-1}$.

Reaction between $\mathbf{5}$ and $\mathbf{N a P z}$, NaSPh, and NaStBu. A typical reaction is shown as follows. In a 100-mL Schlenk flask was added 5 (108 mg, $0.109 \mathrm{mmol}$ ), NaPz (0.30 g, 3.33 $\mathrm{mmol}$ ), and $20 \mathrm{~mL}$ of THF at room temperature. The mixture was then heated under reflux for $4 \mathrm{~h}$ and cooled to room temperature. The solvent was removed under vacuum. $\mathrm{Re}$ crystallization from $\mathrm{CH}_{2} \mathrm{Cl}_{2} /$ hexane afforded the pure product (8) in $85 \%$ yield. Anal. Calcd for $\mathrm{C}_{48} \mathrm{H}_{40} \mathrm{~N}_{4} \mathrm{O}_{4} \mathrm{P}_{2} \mathrm{Ru}_{2}$ : C, 57.59; $\mathrm{H}, 4.02 ; \mathrm{N}, 5.59$. Found: $\mathrm{C}, 57.65 ; \mathrm{H}, 3.91 ; \mathrm{N}, 5.54$. ${ }^{1} \mathrm{H} N M R$ $\left(25^{\circ} \mathrm{C}, 200 \mathrm{MHz}, \mathrm{CDCl}_{3}\right): \mathrm{PPh}_{3}$ at $7.57(\mathrm{br}, 12 \mathrm{H}), 7.39(\mathrm{br}, 18$ $\mathrm{H}) ; \mathrm{H}^{3}$ and $\mathrm{H}^{5}$ on $\mu$-Pz at $6.89(\mathrm{~d}, 2 \mathrm{H}, \mathrm{J}=2.0) ; \mathrm{H}^{4}$ on $\mu$-Pz at $5.78(\mathrm{t}, 1 \mathrm{H}) ; \mathrm{H}^{4}$ on $\mu-\mathrm{Pz}^{\prime}$ at $5.61(\mathrm{~s}, 1 \mathrm{H}) ; \mathrm{Me}^{3}$ and $\mathrm{Me}^{5}$ on $\mu-\mathrm{Pz}^{\prime}$ at $1.56(\mathrm{~s}, 6 \mathrm{H}) .{ }^{31} \mathrm{P}\left\{{ }^{1} \mathrm{H}\right\}$ NMR $\left(25^{\circ} \mathrm{C}, 162 \mathrm{MHz} \mathrm{CDCl}_{3}\right): 12.12$ (s, 2 P). IR $\left(\mathrm{CH}_{2} \mathrm{Cl}_{2}\right)$ : $\mathrm{V}_{\mathrm{co}}, 2028 \mathrm{~s}, 1986 \mathrm{~m}, 1958 \mathrm{~s} \mathrm{~cm}{ }^{-1}$. Compound $\mathbf{9}$ was prepared from $\mathbf{5}$ using $\mathrm{NaSPh}$ in a procedure similar to that used for $\mathbf{8}$. The yield is $87 \%$. Anal. Calcd for $\mathrm{C}_{51} \mathrm{H}_{42} \mathrm{~N}_{2} \mathrm{O}_{4} \mathrm{P}_{2} \mathrm{Ru}_{2} \mathrm{~S}$ : C, 58.72; $\mathrm{H}, 4.05 ; \mathrm{N}, 2.69$. Found: $\mathrm{C}$, 58.76; $\mathrm{H}, 3.94 ; \mathrm{N}, 2.78$. ${ }^{1} \mathrm{H}$ NMR $\left(25^{\circ} \mathrm{C}, 200 \mathrm{MHz}, \mathrm{CDCl}_{3}\right)$ : $\mathrm{PPh}_{3}$ at $7.38(\mathrm{br}, 12 \mathrm{H}), 7.27(\mathrm{~m}, 18 \mathrm{H})$; SPh at $6.81(\mathrm{~m}, 5 \mathrm{H})$; $\mathrm{H}^{4}$ on $\mu-\mathrm{Pz}^{\prime}$ at $5.42(\mathrm{~s}, 1 \mathrm{H})$; $\mathrm{Me}^{3}$ and $\mathrm{Me}^{5}$ on $\mu-\mathrm{Pz}^{\prime}$ at $1.26(\mathrm{~s}$, $6 \mathrm{H}) .{ }^{31} \mathrm{P}\left\{{ }^{1} \mathrm{H}\right\} \mathrm{NMR}\left(25^{\circ} \mathrm{C}, 162 \mathrm{MHz}, \mathrm{CDCl}_{3}\right): 24.66(\mathrm{~s}, 2 \mathrm{P})$. IR $\left(\mathrm{CH}_{2} \mathrm{Cl}_{2}\right) \mathrm{v}_{\mathrm{CO}}, 2016 \mathrm{~s}, 1982 \mathrm{~m}, 1946 \mathrm{~s} \mathrm{~cm}^{-1}$. Compound 10 was prepared from $\mathbf{5}$ using $\mathrm{NaS}^{\mathrm{t}} \mathrm{Bu}$ in a procedure similar to that used for 8. The yield is $90 \%$. Anal. Calcd for $\mathrm{C}_{49} \mathrm{H}_{44^{-}}$ $\mathrm{N}_{2} \mathrm{O}_{4} \mathrm{P}_{2} \mathrm{Ru}_{2} \mathrm{~S}$ : C, 57.53; $\mathrm{H}, 4.53 ; \mathrm{N}, 2.74$. Found: $\mathrm{C}, 57.33 ; \mathrm{H}$, 4.34; $\mathrm{N}, 2.69$. ${ }^{1} \mathrm{H} N M R\left(25^{\circ} \mathrm{C}, 200 \mathrm{MHz}, \mathrm{CDCl}_{3}\right): \mathrm{PPh}_{3}$ at 7.55-7.26 $(\mathrm{m}, 30 \mathrm{H}) ; \mathrm{H}^{4}$ on $\mu-\mathrm{Pz}^{\prime}$ at $5.23(\mathrm{~s}, 1 \mathrm{H}) ; \mathrm{Me}^{3}$ and $\mathrm{Me}^{5}$ on $\mu-\mathrm{Pz}^{\prime}$ at $1.54(\mathrm{br}, 6 \mathrm{H})$; $\mathrm{S}^{\mathrm{t} B u}$ at $1.12(\mathrm{~s}, 9 \mathrm{H})$. ${ }^{31} \mathrm{P}\left\{{ }^{1} \mathrm{H}\right\} \mathrm{NMR}$ $\left(25^{\circ} \mathrm{C}, 162 \mathrm{MHz}_{\mathrm{CDCl}}\right.$ ): 22.46 (d, $1 \mathrm{P}, \mathrm{J}=8.7$ ), 41.9 (d, $1 \mathrm{P}$ ). IR $\left(\mathrm{CH}_{2} \mathrm{Cl}_{2}\right): v_{\mathrm{CO}}, 2004 \mathrm{~s}, 1980 \mathrm{vs}, 1934 \mathrm{~s} \mathrm{~cm}^{-1}$.

Reaction between 3 and dppm. In a 100-mL Schlenk flask was added 3 (133 mg, $0.191 \mathrm{mmol}$ ), dppm (168 mg, 0.438 $\mathrm{mmol}$ ), and $30 \mathrm{~mL}$ of THF at room temperature. The mixture was first stirred at this temperature for $10 \mathrm{~min}$ and then heated under reflux for $28 \mathrm{~h}$, giving a clear orange-red solution. The solvent was removed under vacuum and the resulting solid redissolved in $40 \mathrm{~mL}$ of $\mathrm{CH}_{2} \mathrm{Cl}_{2}$. After filtration through a medium frit, the volume of the filtrate was reduced to ca. 15 $\mathrm{mL}$. A $45 \mathrm{~mL}$ volume of hexane was carefully added on the top of the solution and the two-layer mixture was cooled to $-40{ }^{\circ} \mathrm{C}$ for 1 week, giving orange-red crystals. The crystals were collected on a medium frit and dried in vacuo to give 157 $\mathrm{mg}$ of $\left[\mathrm{Ru}_{2}(\mu-\mathrm{Pz})_{2}(\mathrm{CO})_{2}\left(\mu_{1}, \eta^{2}-\mathrm{dppm}\right)_{2}\right]$ (11), containing a solvate 
Table 2. Crystal Data

\begin{tabular}{|c|c|c|c|c|}
\hline & \multicolumn{4}{|c|}{ compd } \\
\hline & 2 & 5 & 10 & 11 \\
\hline $\begin{array}{l}\text { formula } \\
\text { fw }\end{array}$ & $\begin{array}{l}\mathrm{C}_{21} \mathrm{H}_{26} \mathrm{~N}_{6} \mathrm{O}_{6} \mathrm{Ru}_{2} \\
660.61\end{array}$ & $\begin{array}{l}\mathrm{C}_{47} \mathrm{H}_{40} \mathrm{~N}_{2} \mathrm{O}_{6} \mathrm{P}_{2} \mathrm{Ru}_{2} \\
992.92\end{array}$ & $\begin{array}{l}\mathrm{C}_{49} \mathrm{H}_{46} \mathrm{~N}_{2} \mathrm{O}_{4} \mathrm{P}_{4} \mathrm{Ru}_{2} \mathrm{~S} \\
1023.05\end{array}$ & $\begin{array}{l}\mathrm{C}_{63} \mathrm{H}_{60} \mathrm{Cl}_{2} \mathrm{~N}_{4} \mathrm{O}_{2} \mathrm{P}_{4} \mathrm{Ru}_{2} \\
1302.1\end{array}$ \\
\hline color, habit & yellow, prism & orange, prism & orange, prism & orange, equant \\
\hline diffractometer used & Rigaku AFC6S & Nonius CAD4 & Nonium CAD4 & Siemens SMART-CCD \\
\hline space group & monoclinic, $\mathrm{P} 22_{1} / \mathrm{c}$ & monodinic, $\mathrm{P}_{2} / \mathrm{C}$ & monodinic, $\mathrm{P} 21 / \mathrm{n}$ & monoclinic, $\mathrm{P} 2_{1} / \mathrm{n}$ \\
\hline$a, \AA$ & $14.069(5)$ & $10.4555(16)$ & $13.0086(18)$ & $13.970(3)$ \\
\hline $\mathrm{b}, \AA$ & $13.288(3)$ & $16.113(3)$ & $17.363(3)$ & $25.737(5)$ \\
\hline$c, \AA$ & $14.545(3)$ & $26.063(4)$ & $20.945(3)$ & $16.826(3)$ \\
\hline$\beta$, deq & $98.25(2)$ & $91.420(13)$ & $104.251(12)$ & $92.65(3)$ \\
\hline$V, \AA^{3}$ & 2691(1) & 4389.6(13) & $4585.3(12)$ & $6043(2)$ \\
\hline Z & 4 & 4 & 4 & 4 \\
\hline $\mathrm{D}_{\text {calcd, }} \mathrm{g} \mathrm{cm}^{-3}$ & 1.630 & 1.502 & 1.482 & 1.431 \\
\hline$\lambda(\operatorname{MoK} \alpha), \AA$ & 0.71069 & 0.71069 & 0.71069 & 0.71073 \\
\hline $\mathrm{F}(000)$ & 1320 & 1998 & 2070 & 2656 \\
\hline unit cell detn: no. $2 \theta$ range, deg & $20,8-12$ & $25,17-23$ & $25,17-26$ & whole data \\
\hline scan type & $\omega-2 \theta$ & $\theta-2 \theta$ & $\theta-2 \theta$ & hemisphere \\
\hline $2 \theta$ range, deg & $6-50$ & $2-50$ & $2-50$ & $1-52$ \\
\hline$h, k, I$ range & $16,15, \pm 17$ & $\pm 12,19,30$ & $\pm 15,20,24$ & $\pm 17,31,20$ \\
\hline$\mu(\mathrm{M} \circ \mathrm{K} \alpha), \mathrm{cm}^{-1}$ & 11.67 & 7.89 & 7.57 & 7.40 \\
\hline cryst size, mm & $0.41 \times 0.41 \times 0.66$ & $0.20 \times 0.20 \times 0.30$ & $0.30 \times 0.35 \times 0.35$ & $0.4 \times 0.4 \times 0.4$ \\
\hline temp, $\mathrm{K}$ & 296 & 298 & 298 & 297 \\
\hline no. of measd reflns & 5228 & 4227 & 8063 & 27384 \\
\hline no. of unique reflns & 5014 & 4227 & 8063 & 10543 \\
\hline no. of obsd reflns $\left(\mathrm{N}_{0}\right)$ & $3407(>3 \sigma)$ & $2345(>2 \sigma)$ & $5956(>2 \sigma)$ & $7883(>3 \sigma)$ \\
\hline $\mathbb{R}^{\mathrm{a}}{ }^{\mathrm{a}} \mathrm{R}_{\mathrm{w}}{ }^{\mathrm{a}}$ & $0.047,0.048$ & $0.044,0.041$ & $0.031,0.031$ & $0.038,0.038$ \\
\hline GOFa & 4.00 & 1.08 & 1.28 & 1.70 \\
\hline ofefinement program & TEXSAN & NRCVAX & NRCVAX & SHELXTL-PLUS \\
\hline Eno. of ref params $\left(\mathrm{N}_{\mathrm{p}}\right)$ & 316 & 532 & 542 & 695 \\
\hline weighting scheme & {$\left[\sigma^{2}\left(\mathrm{~F}_{\mathrm{o}}\right)\right]^{-1}$} & {$\left[\sigma^{2}\left(\mathrm{~F}_{\mathrm{o}}\right)+0.0002 \mathrm{~F}_{\mathrm{o}}^{2}\right]^{-1}$} & {$\left[\sigma^{2}\left(\mathrm{~F}_{\mathrm{o}}\right)+0.0001 \mathrm{~F}_{\mathrm{o}}^{2}\right]^{-1}$} & {$\left[\sigma^{2}\left(\mathrm{~F}_{\mathrm{o}}\right)+0.0001 \mathrm{~F}_{\mathrm{o}}^{2}\right]^{-1}$} \\
\hline g $(2$ nd ext coeff $) \times 10 e^{4}$ & & 0 & $0.95(4)$ & $0.000052(8)$ \\
\hline$\Theta(\Delta \rho)_{\max }, \mathrm{e} \AA^{-3}$ & 0.79 & 0.40 & 0.44 & 0.42 \\
\hline$(\Delta \rho)_{\min }, \mathrm{e}^{-3}$ & -1.28 & -0.41 & -0.36 & -0.44 \\
\hline
\end{tabular}

orange, rhombohedron orange, equant

Siemens SMART-CCD Siemens SMART-CCD

triclinic, $\mathrm{P} \overline{1}$

$10.712(4) \quad 14.4764(2)$

$13.939(4) \quad 15.0429(2)$

$17.871(7) \quad 17.0034(1)$

67.156(14) 97.829(1)

$84.40(2) \quad 106.942(1)$

85.273(16) 103.125(1)

2444.5(17) 3367.16(7)

$1.491-1.793$

$0.71073 \quad 0.71073$

$1108 \quad 1796$

whole data whole data

hemisphere hemisphere

3-47 3-51

$\pm 11, \pm 15,19 \quad \pm 17, \pm 17,20$

$7.78 \quad 11.02$

$0.3 \times 0.3 \times 0.4 \quad 0.48 \times 0.45 \times 0.38$

$298 \quad 296$

$9549 \quad 19376$

$6877 \quad 11036$

$5837(>4 \sigma) \quad 11033(>2 \sigma)$

$0.055,0.068 \quad 0.040,0.051$

$1.89 \quad 1.09$

SHELXTL-PLUS SHELXTL-PLUS

556

$\left[\sigma^{2}\left(\mathrm{~F}_{\mathrm{o}}\right)+0.0004 \mathrm{~F}_{\mathrm{o}}^{2}\right]^{-1} \quad\left[\sigma^{2}\left(\mathrm{~F}_{\mathrm{o}}\right)+0.0014 \mathrm{~F}_{\mathrm{o}}^{2}\right]^{-1}$

0

0.72

$-0.77$

$0.0024(2)$

0.94

$-0.60$

999.6

yellow-brown, lamellar orange-brown, irregular Siemens SMART-CCD Siemens P4 orthorhombic, Pbca triclinic, P $\overline{1}$

$17.614(2) \quad 11.744(1)$

$13.624(2) \quad 13.357(1)$

31.294(2) 20.173(2)

$90 \quad 93.19(1)$

$90 \quad 95.09(1)$

$90 \quad 112.90(1)$

7509.6(15) 2889.3(4)

8

$1.768 \quad 1.845$

$0.71073 \quad 0.71073$

$3952 \quad 1524$

whole data $\quad 25,24<2 \theta<25$

hemisphere $\quad \theta-\omega$

3-53 4-45

$20,16,36 \quad 12, \pm 14, \pm 21$

$10.66 \quad 28.96$

$0.61 \times 0.46 \times 0.08 \quad 0.15 \times 0.3 \times 0.4$

296

$32873 \quad 7994$

$6756 \quad 7546$

$4934(>3 \sigma) \quad 4260(>6 \sigma)$

$0.047,0.051 \quad 0.048,0.057$

$1.34 \quad 1.61$

SHELXTL-PLUS SHELXTL-PLUS

497

$\left[\sigma^{2}\left(\mathrm{~F}_{\mathrm{o}}\right)+0.00014 \mathrm{~F}_{\mathrm{o}}{ }^{2}\right]^{-1} \quad\left[\sigma^{2}\left(\mathrm{~F}_{\mathrm{o}}\right)+0.0002 \mathrm{~F}_{\mathrm{o}}{ }^{2}\right]^{-1}$

$0.00027(3) \quad 0.00011(2)$

$0.95 \quad 0.95$

$\begin{array}{ll}0.51 & -0.93\end{array}$

$$
{ }^{a} R=\left[\Sigma|| F_{o}|-| F_{c}|| / \sum \mid F_{o}\right] . \quad R_{w}=\left[\sum w\left(\left|F_{o}\right|-\left|F_{c}\right|\right)^{2} / \Sigma w\left|F_{o}\right|^{2}\right]^{1 / 2} . \quad G O F=\left[\sum w\left(\left|F_{o}\right|-\left|F_{c}\right|\right)^{2} /\left(N_{o}-N_{p}\right)\right]^{1 / 2} .
$$

molecule of $\mathrm{CH}_{2} \mathrm{Cl}_{2}$, which was later confirmed by the elementary analysis results and the ${ }^{1} \mathrm{H}$ NMR spectral evidence. Yield: $63 \%$. Anal. Calcd for $\mathrm{C}_{63} \mathrm{H}_{58} \mathrm{Cl}_{2} \mathrm{~N}_{4} \mathrm{O}_{2} \mathrm{P}_{4} \mathrm{Ru}_{2}$ : C, 58.11;
$\mathrm{H}, 4.64 ; \mathrm{N}, 4.30$. Found: $C, 58.14 ; \mathrm{H}, 4.67 ; \mathrm{N}, 4.40$. ${ }^{1} \mathrm{H} N M R$ $\left(25^{\circ} \mathrm{C}, 200 \mathrm{MHz}, \mathrm{CDCl}_{3}\right)$ : dppm at 7.71-6.99 (m, $\left.40 \mathrm{H}\right), 4.95$ $(\mathrm{m}, 4 \mathrm{H}) ; \mathrm{H}^{4}$ on $\mu-\mathrm{Pz}^{\prime}$ at 5.44 (br, $2 \mathrm{H}$ ); $\mathrm{Me}^{3}$ and $\mathrm{Me}^{5}$ on $\mu-\mathrm{Pz}^{\prime}$ 
at $2.10(\mathrm{~s}, 6 \mathrm{H}), 1.15(\mathrm{~s}, 6 \mathrm{H}) .{ }^{31} \mathrm{P}\left\{{ }^{1} \mathrm{H}\right\} \mathrm{NMR}\left(25^{\circ} \mathrm{C}, 162 \mathrm{MHz}\right.$, $\left.\mathrm{CDCl}_{3}\right): 11.41(\mathrm{~m}, 2 \mathrm{P}),-30.26(\mathrm{~m}, 2 \mathrm{P})$. IR $\left(\mathrm{CH}_{2} \mathrm{Cl}_{2}\right): \mathrm{V}_{\mathrm{CO}}$ $1914 \mathrm{~s}, 1892 \mathrm{~s} \mathrm{~cm}^{-1}$.

Reaction between $\mathbf{1}-\mathbf{3}$ and $\mathbf{N}-\mathbf{N}$ ( $\mathbf{N}-\mathbf{N}=$ bpy, phen). A typical reaction is shown as follows. In a $100-\mathrm{mL}$ Schlenk flask was added $\mathbf{1}$ (205 mg, $0.350 \mathrm{mmol}$ ), bpy (116 mg, 0.742 $\mathrm{mmol}$ ), and $25 \mathrm{~mL}$ of $\mathrm{MeOH}$ at room temperature. The mixture was then heated under reflux for $5 \mathrm{~h}$ and then cooled to room temperature. A $252 \mathrm{mg}$ amount of $\mathrm{NaBPh}_{4}(0.732$ $\mathrm{mmol}$ ) dissolving in $10 \mathrm{~mL}$ of $\mathrm{MeCN}$ was added to the mixture, forming a precipitate within $30 \mathrm{~min}$. The solvents were removed under vacuum. Recrystallization from $\mathrm{CH}_{2} \mathrm{Cl}_{2} / \mathrm{MeOH}$ gave crude product, which was then washed three times each with $5 \mathrm{~mL}$ of $\mathrm{MeOH}$ and $5 \mathrm{~mL}$ of $\mathrm{Et}_{2} \mathrm{O}$ to remove completely the unreacted bpy and dried in vacuo to give $207 \mathrm{mg}$ of pure $\left[\mathrm{Ru}_{2}(\mu-\mathrm{Pz})(\mu-\mathrm{CO})_{2}(\mathrm{CO})_{2}\left(\mu_{1}, \eta^{2}-\mathrm{bpy}\right)_{2}\right]\left[\mathrm{BPh} \mathrm{C}_{4}\right]$ (12) (58\%). Anal. Calcd for $\mathrm{C}_{51} \mathrm{H}_{39} \mathrm{BN}_{6} \mathrm{O}_{4} \mathrm{Ru}_{2}: \mathrm{C}, 60.47 ; \mathrm{H}, 3.88 ; \mathrm{N}, 8.29$. Found: C, $60.27 ; \mathrm{H}, 3.78 ; \mathrm{N}, 8.14 .{ }^{1 \mathrm{H}} \mathrm{NMR}\left(25^{\circ} \mathrm{C}, 200 \mathrm{MHz}\right.$, acetone- $\left.\mathrm{d}_{6}\right)$ : bpy at $10.16(\mathrm{~m}, 4 \mathrm{H}), 8.92(\mathrm{~m}, 4 \mathrm{H}), 8.50(\mathrm{~m}, 4$ $\mathrm{H}), 8.14(\mathrm{~m}, 4 \mathrm{H}) ; \mathrm{BPh}_{4}$ at $7.35(\mathrm{~m}, 8 \mathrm{H}), 6.87(\mathrm{~m}, 8 \mathrm{H}), 6.75$ $(\mathrm{m}, 4 \mathrm{H}) ; \mathrm{H}^{3}$ and $\mathrm{H}^{5}$ on $\mu$-Pz at $5.80(\mathrm{~d}, 2 \mathrm{H}, \mathrm{J}=2.2) ; \mathrm{H}^{4}$ on $\mu$-Pz at $5.29(\mathrm{t}, 1 \mathrm{H})$. IR $\left(\mathrm{CH}_{2} \mathrm{Cl}_{2}\right): \mathrm{v}_{\mathrm{CO}}, 2028 \mathrm{~s}, 1994 \mathrm{w}, 1801$ $\mathrm{w}, 1746 \mathrm{~s} \mathrm{~cm}^{-1}$. A similar reaction between 1 and phen gave $\left[\mathrm{Ru}_{2}(\mu-\mathrm{Pz})(\mu-\mathrm{CO})_{2}(\mathrm{CO})_{2}\left(\mu_{1}, \eta^{2}-\text { phen }\right)_{2}\right]\left[\mathrm{BPh}_{4}\right]$ (13) (68\%). Anal. Calcd for $\mathrm{C}_{55} \mathrm{H}_{39} \mathrm{BN}_{6} \mathrm{O}_{4} \mathrm{Ru}_{2}$ : C, 62.26; $\mathrm{H}, 3.71 ; \mathrm{N}, 7.92$. Eoound: C, 62.03; H, 3.46; N, 8.02. ${ }^{1} \mathrm{H} N M R\left(25^{\circ} \mathrm{C}, 200 \mathrm{MHz}\right.$, acetone- $\left.\mathrm{d}_{6}\right)$ : phen at $10.50(\mathrm{dd}, 4 \mathrm{H}, \mathrm{J}=1.4,5.2), 9.11(\mathrm{dd}, 4$ 要, J = 1.4, 8.2), $8.49(\mathrm{dd}, 4 \mathrm{H}, \mathrm{J}=5.2,8.2), 8.46(\mathrm{~s}, 4 \mathrm{H}) ; \mathrm{BPh}_{4}$ को $7.34(\mathrm{~m}, 8 \mathrm{H}), 6.92(\mathrm{~m}, 8 \mathrm{H}), 6.75(\mathrm{~m}, 4 \mathrm{H}) ; \mathrm{H}^{3}$ and $\mathrm{H}^{5}$ on 造 $\mathrm{Pz}$ at $5.34(\mathrm{~d}, 2 \mathrm{H}, \mathrm{J}=2.0) ; \mathrm{H}^{4}$ on $\mu-\mathrm{Pz}$ at $5.06(\mathrm{t}, 1 \mathrm{H})$. IR $\mathrm{CH}_{2} \mathrm{Cl}_{2}$ ): $\mathrm{v}_{\mathrm{CO}}, 2028 \mathrm{~s}, 1994 \mathrm{w}, 1801 \mathrm{w}, 1746 \mathrm{~s} \mathrm{~cm}^{-1}$. Following æiprocedure similar to that for $\mathbf{1 2}$, the reaction of $\mathbf{2}$ and $\mathbf{3}$ with bpy in the presence of $\mathrm{NaPF}_{6}$ gave only one identical product $\left[\mathrm{Ru}_{2}\left(u-\mathrm{Pz}^{\prime}\right)(\mu-\mathrm{CO})_{2}(\mathrm{CO})_{2}\left(\mu_{1}, \eta^{2}-\mathrm{bpy}_{2}\right]\left[\mathrm{PF}_{6}\right](\mathbf{1 4})\right.$ in 73 and $85 \%$ ypeld, respectively. Anal. Calcd for $\mathrm{C}_{29} \mathrm{H}_{23} \mathrm{~F}_{6} \mathrm{~N}_{6} \mathrm{O}_{4} \mathrm{PRu}_{2}$ : C 40.19; $\mathrm{H}, 2.67 ; \mathrm{N}, 9.69$. Found: $\mathrm{C}, 39.88 ; \mathrm{H}, 2.81 ; \mathrm{N}, 9.80 .{ }^{1} \mathrm{H}$ MMR $\left(25^{\circ} \mathrm{C}, 200 \mathrm{MHz}^{\circ} \mathrm{CDCl}_{3}\right)$ : bpy at $10.24(\mathrm{~m}, 4 \mathrm{H}), 8.67$ (m, $4 \mathrm{H}), 8.38(\mathrm{~m}, 4 \mathrm{H}), 7.95(\mathrm{~m}, 4 \mathrm{H}) ; \mathrm{H}^{4}$ on $\mu$-Pz' at $4.84(\mathrm{~s}$, $\mathrm{H})$; $\mathrm{Me}^{3}$ and $\mathrm{Me}^{5}$ on $\mu-\mathrm{Pz}^{\prime}$ at $3.50(\mathrm{br}, 6 \mathrm{H})$. IR $\left(\mathrm{CH}_{2} \mathrm{Cl}_{2}\right)$ : o, 2027 s, 1992 w, 1800 w, $1743 \mathrm{~s} \mathrm{~cm}^{-1}$. Following a Focedure similar to that for $\mathbf{1 2}$, the reaction of $\mathbf{2}$ and $\mathbf{3}$ with Shen in the presence of $\mathrm{NaPF}_{6}$ gave only one identical product $\left.\mathrm{Ru}_{2}\left(\mu-\mathrm{Pz}^{\prime}\right)(\mu-\mathrm{CO})_{2}(\mathrm{CO})_{2}\left(\mu_{1}, \eta^{2}-\text { phen }\right)_{2}\right]\left[\mathrm{PF}_{6}\right](15)$ in 78 and $80 \%$ xield, respectively. Anal. Calcd for $\mathrm{C}_{33} \mathrm{H}_{23} \mathrm{~F}_{6} \mathrm{~N}_{6} \mathrm{O}_{4} \mathrm{PRu}_{2}$ : C, 43.33; H, 2.53; N, 9.18. Found: C, 43.28; H, 2.60; N, 9.37. ${ }^{1} \mathrm{H}$ MR $\left(25^{\circ} \mathrm{C}, 200 \mathrm{MHz}_{2} \mathrm{CDCl}_{3}\right)$ : phen at $10.65(\mathrm{~d}, 4 \mathrm{H}$, J = 5. 2), $9.20(\mathrm{~d}, 4 \mathrm{H}, \mathrm{J}=8.2), 8.62(\mathrm{dd}, 4 \mathrm{H}, \mathrm{J}=5.2,8.2), 8.54(\mathrm{~s}$, $\$ \mathrm{H}) ; \mathrm{H}^{4}$ on $\mu$-Pz' at $4.78(\mathrm{~s}, 1 \mathrm{H}) ; \mathrm{Me}^{3}$ and $\mathrm{Me}^{5}$ on $\mu$-Pz' at 2.81 (s), $6 \mathrm{H})$. IR $\left(\mathrm{CH}_{2} \mathrm{Cl}_{2}\right): \mathrm{v}_{\mathrm{CO}}, 2026 \mathrm{~s}, 1991 \mathrm{w}, 1801 \mathrm{w}, 1744 \mathrm{~s}$ $\mathrm{Fin}^{-1}$.

Reaction between $\mathrm{Et}_{3} \mathrm{O}^{+} \mathrm{BF}_{4}^{-}$and 1-3. A typical reaction is shown as follows. To the solution of $\mathbf{1}$, prepared by dissolving 1 (100 mg, $0.171 \mathrm{mmol})$ in $1 \mathrm{~mL}$ of $\mathrm{MeCN}$ and 10 $\mathrm{mL}$ of $\mathrm{CH}_{2} \mathrm{Cl}_{2}$ in a $100-\mathrm{mL}$ Schlenk flask, was added with 1 $\mathrm{mL}$ of $\mathrm{Et}_{3} \mathrm{O}^{+} \mathrm{BF}_{4}{ }^{-}$solution $\left(1 \mathrm{M}\right.$ in $\mathrm{CH}_{2} \mathrm{Cl}_{2}$ ). The mixture was then stirred for $1 \mathrm{~h}$ at room temperature, giving a solution IR spectrum with three typical carbonyl stretching bands at 2062 $\mathrm{m}, 2033 \mathrm{~s}$, and $1993 \mathrm{~s} \mathrm{~cm}^{-1}$ for $\left[\mathrm{Ru}_{2}(\mathrm{CO})_{4}(\mathrm{MeCN})_{6}\right]\left[\mathrm{BF}_{4}\right]_{2}{ }^{4}$ which can be converted into $\left[\mathrm{Ru}_{2}(\mathrm{CO})_{4}(\mathrm{MeCN})_{4}\left(\mathrm{PPh}_{3}\right)_{2}\right]\left[\mathrm{BF}_{4}\right]_{2}$ by following the established procedure. ${ }^{4 a}$ Yield: $90 \%$.

Reaction between $\mathrm{Et}_{3} \mathrm{O}^{+} \mathrm{BF}_{4}^{-}$and 4-6. A typical reaction is shown as follows. To the solution of $\mathbf{4}$, prepared by dissolving 5 (100 $\mathrm{mg}, 0.101 \mathrm{mmol}$ ) in $10 \mathrm{~mL}$ of $\mathrm{CH}_{2} \mathrm{Cl}_{2}$ and 1 $\mathrm{mL}$ of $\mathrm{MeCN}$ in a $100-\mathrm{mL}$ Schlenk flask, was added with 0.5 $\mathrm{mL}$ of $\mathrm{Et}_{3} \mathrm{O}^{+} \mathrm{BF}_{4}{ }^{-}$solution ( $1 \mathrm{M}$ in $\mathrm{CH}_{2} \mathrm{Cl}_{2}$ ). The mixture was then stirred for $2 \mathrm{~h}$ at room temperature, and $5 \mathrm{~mL}$ of $\mathrm{MeOH}$ was added to decompose unreacted $\mathrm{Et}_{3} \mathrm{O}^{+} \mathrm{BF}_{4}{ }^{-}$. Volatile substance was removed under vacuum. Recrystallization from
$\mathrm{CH}_{2} \mathrm{Cl}_{2} / \mathrm{MeOH}$ gave $105 \mathrm{mg}$ of pure solid. It was identified as $\left[\mathrm{Ru}_{2}(\mathrm{CO})_{4}(\mathrm{MeCN})_{4}\left(\mathrm{PPh}_{3}\right)_{2}\right]\left[\mathrm{BF}_{4}\right]_{2}$ by comparing the spectral evidences with those reported. ${ }^{4}$ Yield: $88 \%$.

Reaction between 4 and $I_{2}$. In a $100-\mathrm{mL}$ Schlenk flask was added 4 ( $107 \mathrm{mg}, 0.110 \mathrm{mmol}$ ) and $5 \mathrm{~mL}$ of $\mathrm{CH}_{2} \mathrm{Cl}_{2}$ at room temperature. This solution was then added dropwise with a $\mathrm{CH}_{2} \mathrm{Cl}_{2}$ solution of $\mathrm{I}_{2}$, prepared by dissolving 2 equiv of $\mathrm{I}_{2}$ (ca. $0.060 \mathrm{~g}$ ) in $5 \mathrm{~mL}$ of $\mathrm{CH}_{2} \mathrm{Cl}_{2}$. The mixture was stirred for 0.5 $\mathrm{h}$, and the solvent was then removed under vacuum. Recrystallization from $\mathrm{CH}_{2} \mathrm{Cl}_{2} / \mathrm{MeOH}$ afforded $0.108 \mathrm{~g}$ of the pure product $\left[\mathrm{Ru}_{2}(\mu-\mathrm{Pz})_{2}(\mu-\mathrm{I})(\mathrm{CO})_{4}\left(\mathrm{PPh}_{3}\right)_{2}\right]\left[\mathrm{I}_{3}\right]$ (16) in $73 \%$ yield. Anal. Calcd for $\mathrm{C}_{46} \mathrm{H}_{36}{ }_{4} \mathrm{~N}_{4} \mathrm{O}_{4} \mathrm{P}_{2} \mathrm{Ru}_{2}$ : C, 37.32; $\mathrm{H}, 2.45 ; \mathrm{N}, 3.78$. Found: C, 37.56; $\mathrm{H}, 2.54 ; \mathrm{N}, 3.92$. ${ }^{1} \mathrm{H}$ NMR $\left(25^{\circ} \mathrm{C}, 200 \mathrm{MHz}\right.$, acetone- $\left.\mathrm{d}_{6}\right): \mathrm{PPh}_{3}$ at $7.79-7.53(\mathrm{~m}, 30 \mathrm{H}) ; \mathrm{H}^{3}$ and $\mathrm{H}^{5}$ on $\mu-\mathrm{Pz}$ at $6.99(\mathrm{~d}, 4 \mathrm{H}, \mathrm{J}=2.3) ; \mathrm{H}^{4}$ on $\mu$-Pz at $5.74(\mathrm{t}, 2 \mathrm{H}) .{ }^{31} \mathrm{P}\left\{{ }^{1} \mathrm{H}\right\}$ NMR $\left(25^{\circ} \mathrm{C}, 162 \mathrm{MHz}\right.$, acetone- $\left.\mathrm{d}_{6}\right)$ : 38.74 (s, $\left.2 \mathrm{P}\right)$. IR: $\mathrm{v}_{\mathrm{CO}}$, $2072 \mathrm{~s}, 2024 \mathrm{~s} \mathrm{~cm}^{-1}$ in $\mathrm{CH}_{2} \mathrm{Cl}_{2} ; \mathrm{v}_{\mathrm{CO}}, 2068 \mathrm{~s}, 2016 \mathrm{~s} \mathrm{~cm}^{-1}$ in $\mathrm{KBr}$.

Single-Crystal X-ray Diffraction Studies of 2, 5, 10 12, and 14-16. Suitable single crystals were grown from $\mathrm{CH}_{2-}$ $\mathrm{Cl}_{2} /$ hexane or $\mathrm{CH}_{2} \mathrm{Cl}_{2} / \mathrm{Et}_{2} \mathrm{O}$ at room temperature to do the single-crystal structure determination. The X-ray diffraction data for 2, 5, 10, and $\mathbf{1 6}$ were measured on a four-circle diffractometer, and those for 11, 12, 14, and 15 were measured in frames with increasing $\omega\left(0.3^{\circ} /\right.$ frame $)$ and with the scan speed at $10.00 \mathrm{~s} /$ frame on a Siemens SMART-CCD instrument, equipped with a normal focus and $3 \mathrm{~kW}$ sealed-tube $X$-ray source. For data collected on the four-circle diffractometer, three standard reflections were monitored every $1 \mathrm{~h}$ or every 50 reflections throughout the collection. The variation was less than 2\%. Empirical absorption corrections were carried out on the basis of an azimuthal scan. For 2, the structure was solved by direct methods and refined by a full-matrix least-squares procedure using TEXSAN. ${ }^{8}$ For $\mathbf{5}$ and 10, the structures were solved by heavy-atom method and refined by a full-matrix least-squares procedure using NRCVAX. ${ }^{9} \mathrm{~F}$ or $\mathbf{1 1}$, 12, and 14-16, the structures were solved by direct methods and refined by a full-matrix least-squares procedure using SHELXTL-PLUS. ${ }^{10}$ The other essential details of singlecrystal data measurement and refinement are given in Table 2. One molecule of $\mathrm{CH}_{2} \mathrm{Cl}_{2}$ was found in the asymmetric unit of the crystals used for $\mathbf{1 1}, \mathbf{1 2}$, and $\mathbf{1 4}$, whereas one and a half molecules of $\mathrm{CH}_{2} \mathrm{Cl}_{2}$ were located for $\mathbf{1 6}$. The solvent hydrogen positions in this structure were not included in the structure refinement.

Acknowledgment. Financial support for this work by the National Science Council of Republic of China (Contract NSC85-2113-M 006-012) is gratefully acknowledged.

Supporting Information Available: An ORTEP plot for 14B (Figure 9) and tables of non-hydrogen atomic coordinates and equivalent isotropic displacement coefficients, complete bond lengths and angles, anisotropic displacement coefficients, and hydrogen coordinates and B values for $\mathbf{2 ,} \mathbf{5}, \mathbf{1 0}-\mathbf{1 2}$, and 14-16 (58 pages). Ordering information is given on any current masthead page.

\section{OM9600708}

(8) Crystal Structure Analysis Package, Molecular Structure Corp., The Woodlands, TX, 1985, 1992.

(9) Gabe, E. J .; Le page, Y.; Charland, J .-P.; Lee, F. L.; White, P. S. J . Appl. Crystallogr. 1989, 22, 384.

(10) (a) Sheldrick, G. M. SHELXTL-Plus Crystallographic System, release 4.21: Siemens Analytical X-ray Instruments: Madison, WI, 1991. (b) Siemens Analytical X-ray Instruments Inc., Karlsruhe, Germany, 1991. 